Ann. Sci. forest., 1973, 30 (1), 31-62

\title{
CARACTÉRISTIQUES DU BOIS DE PIN MARITIME VARIABILITÉ ET TRANSMISSION HÉRÉDITAIRE
}

\author{
R. KELLER \\ Station de Recherches sur la Qualité des Bois. \\ Centre National de Recherches forestières, I.N.R.A., \\ Champenoux 54370 Einville
}

\section{RÉSUMÉ}

La qualité du bois de pin maritime peut être appréciée à l'aide des mesures de nombreuses caractéristiques. Dans cette étude, $\pi$ 'ont été envisagées que celles qui faisaient appel à des procédés non destructifs, essentiellement des sondages à la tarière de $5 \mathrm{~mm}$.

L'échantillonnage a une double origine : des arbres adultes (arbres-mères) dispersés dans le massif landais et des descendants issus de graines récoltées sur ces derniers (demi-frères du test D 441) rassemblés dans un dispositif en blocs incomplets équilibrés.

Sur les arbres-mères, on a mesuré des caractéristiques densitométriques : densité globale et composantes de la densité relatives aux vingt années s'échelonnant de 11 à 30 ans, une caractéristique de croissance : la largeur des cernes, deux caractéristiques papetières : rendement en fibres et longueur des fibres, une caractéristique morphologique : l'angle du fil du bois dans les couches extérieures avec l'axe de l'arbre (angle de la fibre torse). On a étudié également, lorsque c'était possible, l'anisotropie de ces données entre le côté est et le cóté ouest.

Les mêmes mesures, adaptées à la taille des échantillons, ont été faites sur les demi-frères du test D 441 ; il faut $\mathrm{y}$ ajouter le rendement en résine.

Dans le cas des arbres-mères, il ressort que l'hétérogénéité du bois mesurée par l'écart entre les valeurs de la densité maximale et de la densité minimale est une caractéristique intéressante pour différencier les individus. Les largeurs de cernes, en revanche, ne fournissent aucun moyen de discrimination. L'anisotropie se manifeste dans le cas de nombreuses données, mais est cependant inexistante lorsqu'on considère certains niveaux de densité élevée.

Les résultats du test D 441 portent sur des caractères de bois juvénile en évolution d'année en année, à densité faible, et dont l'hétérogénéité augmente du fait que les valeurs des densités maximales croissent plus rapidement que celles des densités minimales. La variabilité des résultats à l'intérieur des familles, ou encore en fonction du temps, est souvent grande et empêche de donner des conclusions définitives. L'anisotropie existe dès les premières années de la vie des jeunes arbres, sauf en ce qui concerne les zones du bois final des deux saisons de végétation étudiées. Des différences d'anisotropie existent entre les familles en ce qui concerne la densité globale.

Des calculs d'héritabilité ont été faits à partir de deux méthodes : analyse de variance sur les familles des descendants et corrélation entre les arbres-mères et les demi-frères. Dans le premier cas, les composantes de la densité, et notamment l'écart entre la densité maximale et la densité minimale, se sont révélées être particulièrement héritables. Dans le second cas, les calculs de corrélation n'ont mis en évidence d'héritabilités que pour une densité minimale, l'infradensité, et un rendement en fibres,

Enfin, des calculs généraux de corrélation portent sur toutes les données disponibles du test D 441, des arbres-mères et sur les couples de résultats des familles de descendants avec leurs parents respectifs. 


\section{INTRODUCTION}

L'amélioration du pin maritime (Pinus pinaster Ait) est à l'origine de nombreuses études de production et, de plus en plus, de qualité du bois. Cette essence a une grande importance, non seulement en France et pour certains de ses voisins, le Portugal notamment, mais encore pour des pays qui, comme l'Australie, l'utilisent dans leurs reboisements.

La notion de qualité du bois vient prendre place maintenant naturellement parmi les préoccupations de ceux qui, à des titres divers, s'intéressent aux essences ligneuses. De nombreux facteurs de cette qualité peuvent être étudiés; entre autres exemples, on trouve dans les travaux effectués en Australie (Nicholıs, Perry et Hopkins, 1968) sur la sélection de pins maritimes d'origine portugaise des références à des caractéristiques telles que la largeur des cernes, l'angle de la fibre torse, la densité du bois, la longueur des trachéides et le pourcentage de bois à forte densité estimé par le pourcentage de bois d'été; ces données sont couramment envisagées, mais il est possible d'en retenir d'autres.

La qualité du bois peut évidemment ètre étudiée sur les individus adultes, lorsqu'ils sont abattus, mais on ne peut attendre de cette méthode qu'elle serve dans l'orientation de la sélection, étant donné la lenteur du renouvellement des générations chez les arbres. C'est une des raisons pour lesquelles les recherches actuelles portent aussi sur de jeunes sujets chez lesquels sont mesurés les caractères de la matière ligneuse. Il se trouve, et c'est là une difficulté sérieuse, que ces derniers sont dans un état " juvénile » évoluant au fur et à mesure que l'arbre vieillit et tend vers un état adulte; à ce stade, les variations des caractères seraient plutôt dues aux influences du milieu.

Dans l'état actuel des connaissances, il n'est pas très facile d'estimer (ILly, 1966) dans quelle mesure les caractères d'arbres parents adultes se retrouvent chez leurs descendants, ou inversement. S'il est possible de calculer les corrélations phénotypiques entre un état jeune et un état adulte chez un même individu par analyse de tige en particulier, il ne sera pas possible de connaître les corrélations génétiques entre ces états tant que les plantations actuelles destinées à éliminer l'action du milieu ne seront pas suffisamment âgées.

L'étude génétique des caractères des arbres forestiers est faite généralement à l'aide de I'évaluation de coefficients, ou héritabilités, par analyse de variance aboutissant à la corrélation intraclasse, qui chiffrent la parenté entre les individus issus d'une même famille vis à vis de l'ensemble des familles d'individus croissant dans les mêmes conditions (au sein d'une mème plantation comparative), ou encore plus directement, l'intensité du lien existant entre descendants d'une famille et arbre-mère à l'origine de cette famille, évaluée à partir de la pente de la droite de régression représentant les variations des moyennes des valeurs des descendances en fonction de celles des parents (arbres-mères); dans ce dernier cas, en quelque sorte, cette évaluation est faite malgré l'influence du milieu car, si les enfants sont regroupés dans un même lieu, les arbres-mères, eux, sont dispersés dans un vaste massif forestier où les variations du milieu et de la concurrence sont importantes.

On pourrait aussi, dans le cadre de telles études, « rejuvéniliser » les arbres-mères afin de comparer les clones obtenus aux descendants de ces mêmes parents issus de graines. La rejuvénilisation est provoquée par l'intermédiaire de la greffe qui redonne aux greffons prélevés sur les arbres-mères des caractẻres de jeunesse, la multinodalité qui réapparait après 
quelques années en est d'ailleurs une manifestation, identique à ce que l'on peut observer sur des semis jeunes dont la croissance annuelle en hauteur se traduit par l'apparition de plus d'un verticille par saison de végétation.

\section{1. - ORIGINE DES ÉCHANTILLONS}

L'étude de ces pins maritimes a été faite essentiellement à partir de carottes de sondage de $5 \mathrm{~mm}$ de diamètre obtenues à l'aide de tarières de Pressler; les prélèvements ont été effectués sur deux types d'arbres :

\section{1. - Arbres-mères}

Les arbres-mères sont dispersés dans tout le massif landais (Illy, 1966 op. cit.); il en subsistait 31 en 1967. Pour éviter la zone de cicatrisation des cares, ces arbres ont été sondés à $4 \mathrm{~m}$ de hauteur. Une carotte droite et une carotte oblique ont été prises sur chaque individu.

\section{2. - Demi-frères}

Les demi-frères du test D $441^{\text {(1) }}$ descendent de ces arbres-mères; 41 familles sont regroupées à l'Hermitage dans un dispositif en blocs incomplets équilibrés représentant 40 descendances de 40 arbres-mères et 1 témoin constitué d'arbres issus d'un mélange de graines du commerce. Ces 40 descendances maternelles représentent les 31 descendances des arbres cités ci-dessus, plus 9 descendances d'arbres abattus avant 1967.

Les arbres du test D 441 avaient été plantés à l'âge de 1 an en automne 1962; Ies prélèvements ont été faits sur eux en juin 1967, soit sur des individus d’à peine 6 ans. Comme pour les arbres-mères, des prélèvements droits et des prélèvements obliques ont été effectués, les premiers sur 10 individus par famille et les seconds sur 10 autres individus; les arbres sondés ont été choisis uniquement en fonction de leur emplacement dans les parcelles unitaires du dispositif; toutes les fois que cela a été possible, il s'est agi des premiers et deuxièmes arbres NordOuest de la ligne Nord de chaque parcelle.

Étant donné l'âge de ces arbres, les caractéristiques mesurées seront celles d'un bois juvénile encore bien différent du bois adulte des arbres-mères.

\section{2. - MESURES - RÉSULTATS}

\section{1. - Arbres-mères}

\subsection{Densité globale.}

L'infradensité a êté déterminée sur tous les échantillons selon la méthode classique de la saturation intégrale utilisée dans notre laboratoire (POLGE, 1963); cette caractéristique dépend de l'orientation du trou de sondage dans le tronc. L'anisotropie du pin maritime dans les

(1). Il s'agit d'une plantation comparative de descendances maternelles installée par l'unité « amélioration des arbres forestiers \& de la Station de recherches forestières de Bordeaux, dans le cadre du programme de sélection génétique du pin maritime. 
Landes étant la plupart du temps marquée entre les côtés est et ouest, on a mesuré les densités du bois suivant ces deux directions. Généralement, le bois situé du côté est est plus dense que celui qui se trouve du côté ouest, ce dernier étant le plus souvent exempt de bois de compression.

Pour des raisons de commodité, on a reporté dans le tableau 1 les équivalences entre la numérotation des familles (nombres à 4 chiffres) et leur code utilisé par la suite dans les autres tableaux (nombres à 2 chiffres).

\section{TABLEAU 1 - TABLE 1}

Tableau d'équivalence entre les origines des familles: nombre à 4 chiffres, et leur code : nombre à I ou 2 chiffres.

Table of equivalence between the families (4 numbers) and sheir codes ( $I$ or 2 numbers).

\begin{tabular}{|c|c|c|c|}
\hline Famille & Code & Code & Famille \\
\hline $\begin{array}{l}0001 \\
0002 \\
0003 \\
0005 \\
0007 \\
0008 \\
0010 \\
0011 \\
0013 \\
0014 \\
0101 \\
0137 \\
0147 \\
0150 \\
0158 \\
0205 \\
0248 \\
0250 \\
0251 \\
0252 \\
0281 \\
0282 \\
0283 \\
1301 \\
1303 \\
1305 \\
1306 \\
1307 \\
1308 \\
2802 \\
3102 \\
5101 \\
5102 \\
5301 \\
5302 \\
5303 \\
5305 \\
5306 \\
5307 \\
5308 \\
\text { Témoin }\end{array}$ & $\begin{array}{r}32 \\
15 \\
33 \\
14 \\
17 \\
37 \\
30 \\
35 \\
26 \\
23 \\
10 \\
27 \\
5 \\
36 \\
7 \\
3 \\
2 \\
34 \\
16 \\
12 \\
9 \\
4 \\
40 \\
11 \\
24 \\
20 \\
21 \\
18 \\
39 \\
6 \\
28 \\
19 \\
22 \\
1 \\
29 \\
8 \\
38 \\
13 \\
25 \\
31 \\
41\end{array}$ & $\begin{array}{l}1 \\
2 \\
3 \\
4 \\
5 \\
6 \\
7 \\
8 \\
9 \\
10 \\
11 \\
12 \\
13 \\
14 \\
15 \\
16 \\
17 \\
18 \\
19 \\
20 \\
21 \\
22 \\
23 \\
24 \\
25 \\
26 \\
27 \\
28 \\
29 \\
30 \\
31 \\
32 \\
33 \\
34 \\
35 \\
36 \\
37 \\
38 \\
39 \\
40 \\
41\end{array}$ & $\begin{array}{l}5301 \\
0248 \\
0205 \\
0282 \\
0147 \\
2802 \\
0158 \\
5303 \\
0281 \\
0101 \\
1301 \\
0252 \\
5306 \\
0005 \\
0002 \\
0251 \\
0007 \\
1307 \\
5101 \\
1305 \\
1306 \\
5102 \\
0014 \\
1303 \\
5307 \\
0013 \\
0137 \\
3102 \\
5302 \\
0010 \\
5308 \\
0001 \\
0003 \\
0250 \\
0011 \\
0150 \\
0008 \\
5305 \\
1308 \\
0283 \\
\text { Témoins }\end{array}$ \\
\hline
\end{tabular}


Le tableau 2 donne, pour les 31 arbres-mères subsistant en 1967, les valeurs de la densité pour les carottes droites et obliques, les côtés est et ouest, la carotte complète et la carotte limitée aux 20 cernes de la période de la vie de l'arbre s'étendant de 11 à 30 ans.

La densité du bois des 20 cernes allant de 11 à 30 ans est légèrement supérieure à la densité de l'ensemble de la carotte; elle vaut $484 \mathrm{~g} / \mathrm{dm}^{3}$ pour le côté est et $481 \mathrm{~g} / \mathrm{dm}^{3}$ pour le côté ouest.

\subsection{Composantes de la densité des 20 années dans la vie de l'arbre, allant de $1 /$ à 30 ans.}

Les courbes de l'évolution de la densité du bois de 33 arbres-mères ont été étudiées de façon à déterminer si les caractéristiques que l'on peut y trouver sont de bons critères capables de faire distinguer au mieux des individus.

On a mesuré huit pourcentages $\left(\% 1 d>x_{\mathrm{g}} / \mathrm{dm}^{3}\right)$ définissant les proportions de bois de densité supérieure à $300 \mathrm{~g} / \mathrm{dm}^{3}$ jusqu'à $1000 \mathrm{~g} / \mathrm{dm}^{3}$ par échelons de $100 \mathrm{~g} / \mathrm{dm}^{3}$. Les densités de valeurs extrêmes : densités minimales et densités maximales ont été déterminées parce que l'on pense que la différence entre ces grandeurs, ou écart maximum de densité, est un facteur qui caractérise bien I'hétérogénéité du bois.

Le tableau 3 regroupe ces données auxquelles on a joint la largeur des cernes et l'infradensité de la période considérée.

Généralement, les arbres ont une proportion très élevée de bois dont la densité dépasse $300 \mathrm{~g} / \mathrm{dm}^{3}$. On trouve en effet que le pourcentage atteint $95,9 \%$ en moyenne $(97,4 \%$ pour l'est et $94,4 \%$ pour l'ouest (*).

Le pourcentage de bois de densité supérieure à $400 \mathrm{~g} / \mathrm{dm}^{3}$ vaut en moyenne $58,7 \%$. Certains arbres ont encore une forte proportion de leur bois dont la densité dépasse ce chiffre.

Les deux niveaux suivants : $500 \mathrm{~g} / \mathrm{dm}^{3}$ et $600 \mathrm{~g} / \mathrm{dm}^{3}$, présentent des pourcentages décroissants et dans l'ordre on a trouvé $39,6 \%$ et $29,9 \%$. Dans un essai de différenciation maximum des arbres par analyse factorielle, il a semblé que ces deux niveaux jouaient un rôle particulier dans le cas des côtés est et ouest; en effet, ils sont très liés à certaines des variables synthétiques qui sont apparues dans ces calculs, dont le principe est bien connu et que donc nous ne détaillerons pas ici.

Dans le cas du côté est, aussi bien le pourcentage au niveau $500 \mathrm{~g} / \mathrm{dm}^{3}$ que le pourcentage au niveau $600 \mathrm{~g} / \mathrm{dm}^{3}$ sont liés avec un coefficient de corrélation $\mathrm{r}=0,94$ à la variable synthétique qui rend compte de $46,4 \%$ de la variabilité entre les arbres-mères.

Dans le cas du côté ouest, on observe le même phénomène en ce qui concerne le niveau $600 \mathrm{~g} / \mathrm{dm}^{3}$ qui est lié, par son coefficient de corrélation $\mathrm{r}=0,90$ avec la variable synthétique qui prend en compte $48,8 \%$ de la variabilité entre les arbres-mères.

Si l'on considère la moyenne est + ouest, c'est une autre caractéristique plus classique qui apparaît : la densité globale des 20 années allant de 11 à 30 ans.

Les niveaux supérieurs à $600 \mathrm{~g} / \mathrm{dm}^{3}$ ont des ; ourcentages régulièrement décroissants de 22,3 dans le cas des $700 \mathrm{~g} / \mathrm{dm}^{3}$, à 13,1 dans celui $\mathrm{c}: ; 800 \mathrm{~g} / \mathrm{dm}^{3}, 5,0$ pour $900 \mathrm{~g} / \mathrm{dm}^{3}$, et enfin 0,4 pour le niveau des $1000 \mathrm{~g} / \mathrm{dm}^{3}$; ces niveaux n'ont pas joué de rôle particulier dans les

(*) Conventionnellement, on notera l'est par (E) et l'ouest par (W) dans ce qui suit. 
TABLEAU 2 - TABle 2

Mesures sur les arbres-mères, Measurements of the mother-trees.

arbres-mères - mother-trees, $\mathrm{E}=$ east $\mathrm{W}=$ west, densité - mean specific gravity, caur - pith side, ecorce - bark side.

carotte droite = normal increment boring.

carotte oblique - slanting increment boring. cerne = annual ring,

pourcentage de resine = percentage of resin,

longueur de fibres $=$ fiber length,

rendement en fibres = pulp yield,

angle de la fibre torse $=$ spiral grain .

\begin{tabular}{|c|c|c|c|c|c|c|c|c|c|c|c|c|c|c|c|c|c|}
\hline \multirow[b]{3}{*}{ Code } & \multicolumn{6}{|c|}{ Densité } & \multirow{2}{*}{\multicolumn{2}{|c|}{$\begin{array}{l}\text { Pourcentage } \\
\text { de résine }\end{array}$}} & \multirow{2}{*}{\multicolumn{2}{|c|}{$\begin{array}{l}\text { Longueur de } \\
\text { fibres-Écorce }\end{array}$}} & \multirow{2}{*}{\multicolumn{2}{|c|}{$\begin{array}{l}\text { Longueur de } \\
\text { fibres-Ceeur }\end{array}$}} & \multirow{2}{*}{\multicolumn{2}{|c|}{$\begin{array}{l}\text { Rendement en } \\
\text { fibres-Écorce }\end{array}$}} & \multirow{2}{*}{\multicolumn{2}{|c|}{$\begin{array}{l}\text { Rendement en } \\
\text { fibres-Caxur }\end{array}$}} & \multirow{3}{*}{$\begin{array}{l}\text { Angle } \\
\text { de la } \\
\text { fibre } \\
\text { torse } \\
\text { T'oues }\end{array}$} \\
\hline & \multicolumn{2}{|c|}{$\begin{array}{l}\text { Carur + Écorce } \\
\text { Carottes droites }\end{array}$} & \multicolumn{2}{|c|}{$\begin{array}{l}\text { Carur + Ecorce } \\
\text { Carottes obliques }\end{array}$} & \multicolumn{2}{|c|}{$\begin{array}{c}20 \text { cernes } \\
\text { de } 11 \text { à } 30 \text { ans } \\
\text { Carottes droites }\end{array}$} & & & & & & & & & & & \\
\hline & E & w & E & w & E & w & E & w & $\mathrm{E}$ & w & E & w & E & w & E & w & \\
\hline 1 & 414 & 384 & 426 & 388 & 413 & 380 & 9,45 & 3,41 & 3,76 & 4,36 & 3,15 & 3,87 & 43 & 52 & 46 & 45 & 4 \\
\hline 3 & 413 & 408 & 428 & 398 & 404 & 391 & 4,97 & 4,83 & 4,08 & 4,25 & 3,87 & 3,74 & 51 & 51 & 50 & 46 & 0 \\
\hline 5 & 446 & 401 & 464 & 410 & 461 & 404 & 6,86 & 3,45 & 3,52 & 4,50 & 3,40 & 4,49 & 49 & 58 & 49 & 48 & 4 \\
\hline 6 & 421 & 413 & 427 & 398 & 387 & 403 & 5,26 & 7,46 & 4,03 & 4,47 & 3,08 & 3,47 & 49 & 55 & 58 & 53 & 0 \\
\hline 7 & 404 & 379 & 399 & 394 & 414 & 382 & 3,84 & 3,84 & 3,82 & 3,68 & 3,27 & 3,33 & 52 & 45 & 49 & 46 & 1 \\
\hline 8 & 418 & 394 & 409 & 383 & 417 & 385 & 5,08 & 2,23 & 3,20 & 3,39 & 2,87 & 3,13 & 46 & 45 & 45 & 45 & 3 \\
\hline 9 & 483 & 464 & 495 & 427 & 471 & 435 & 4,44 & 6,25 & 4,04 & 3,70 & 3,02 & 2,89 & 51 & 47 & 56 & 48 & 7 \\
\hline 10 & 431 & 397 & 455 & 413 & 445 & 412 & - & - & 4,55 & 4,03 & 4,23 & 3,77 & 53 & 57 & 51 & 60 & -2 \\
\hline 13 & 401 & 390 & 414 & 396 & 420 & 398 & 6,90 & 6,96 & 3,22 & 4,01 & 3,45 & 2,77 & 51 & 49 & 55 & 44 & 3 \\
\hline
\end{tabular}


TABLEAU 3 - TABLE 3

Mesures sur les arbres-mères : 20 cernes (de 11 à 30 ans). Measurements of 20 annual growth increments of the mother-trees.

largeur de cernes $=$ ringwidih,

$\mathrm{d}=$ average of the minimal annual densities,

$\mathrm{D}=$ average of the maximal annual densities.

$\% 1 \mathrm{~d}>\times g / \mathrm{dm}^{2}-$ percentage of wood with a density $>\times g / d m^{3}$

rang $=$ order .

Chaque arbre de ce tableau est caractérisé par deux lignes de résultats : la premièré est relative au côté est (E) et la seconde, au côté ouest (W). ?

Each tree of this table is characterized by 2 lines of results : the first one concerns the east side (E) and the second, the west side (W).

\begin{tabular}{|c|c|c|c|c|c|c|c|c|c|c|c|c|c|c|c|c|}
\hline Code & $\begin{array}{l}\text { Largeur } \\
\text { des } \\
\text { cernes }\end{array}$ & $\begin{array}{l}\text { Infra- } \\
\text { densité }\end{array}$ & $\begin{array}{c}\stackrel{d}{11 \mathrm{a}} \\
30 \text { ans }\end{array}$ & $\begin{array}{c}\text { D } \\
11 \text { à } \\
30 \text { ans }\end{array}$ & $\begin{array}{l}\mathrm{D}_{11 \mathrm{a}} d \\
30 \text { ans }\end{array}$ & $\begin{array}{c}\%, l d \geqslant \\
300 \\
\mathrm{~g} / \mathrm{dm}^{3}\end{array}$ & $\begin{array}{c}\% l d \geqslant \\
400 \\
\mathrm{~g} / \mathrm{dm}^{3}\end{array}$ & $\begin{array}{c}\% l d l \\
500 \\
\mathrm{~g} / \mathrm{dm}^{3}\end{array}$ & $\begin{array}{c}\% l d l \\
600 \\
\mathrm{~g} / \mathrm{dm}^{3}\end{array}$ & $\begin{array}{c}\% \frac{d d}{700} \geqslant \\
\mathrm{~g} / \mathrm{dm}^{3}\end{array}$ & $\begin{array}{c}\% l d \geqslant \\
800 \\
\mathrm{~g} / \mathrm{dm}^{2}\end{array}$ & $\begin{array}{c}\% / d \geqslant \\
900 \\
g / d^{2}\end{array}$ & $\begin{array}{c}\% / d \geqslant \\
1000 \\
\mathrm{~g} / \mathrm{dm}^{3}\end{array}$ & $\begin{array}{c}\text { Angle de } \\
\text { la fibre } \\
\text { torse }\end{array}$ & \multicolumn{2}{|c|}{$\begin{array}{l}\text { Rang pour } \\
\text { D } \frac{d}{30} \text { ans }\end{array}$} \\
\hline 1 & 6,39 & 413 & 327 & 769 & 442 & 99,8 & 60,9 & 42,9 & 30 & 14,3 & 1,6 & 0 & 0 & \multirow{3}{*}{4} & \multicolumn{2}{|l|}{31} \\
\hline 3 & $\begin{array}{l}3,70 \\
3,79\end{array}$ & $\begin{array}{l}380 \\
404\end{array}$ & $\begin{array}{l}312 \\
326\end{array}$ & $\begin{array}{l}886 \\
884\end{array}$ & $\begin{array}{l}574 \\
558\end{array}$ & $\begin{array}{l}99 \\
99,3\end{array}$ & $\begin{array}{l}35,2 \\
51,1\end{array}$ & $\begin{array}{l}33,2 \\
36,6\end{array}$ & $\begin{array}{l}24,4 \\
26,2\end{array}$ & $\begin{array}{l}17,7 \\
19.1\end{array}$ & 10,1 & 1,6 & 0 & & \multirow{2}{*}{\multicolumn{2}{|c|}{11}} \\
\hline & 4,60 & 391 & 333 & 860 & 527 & $100^{\circ}$ & 55,7 & 35,5 & 26,2 & $\begin{array}{l}19,1 \\
18,2\end{array}$ & 10 & $\begin{array}{l}2,1 \\
0,9\end{array}$ & $\begin{array}{l}0 \\
0\end{array}$ & & & \\
\hline 5 & 3,75 & 461 & 391 & 921 & 530 & 100 & 84,6 & 51,4 & 39,1 & 29,6 & 20,9 & 9,9 & 0,4 & & \multicolumn{2}{|l|}{21} \\
\hline & 3,90 & 404 & 336 & 836 & 500 & 98,2 & 55,1 & 37,3 & $27^{\circ}$ & 17,8 & 6 & 1 & 0,3 & 4 & \multirow{2}{*}{\multicolumn{2}{|c|}{18}} \\
\hline 6 & 3,80 & 387 & 297 & 834 & 537 & 88,3 & 44,7 & 35,5 & 27,9 & 17,6 & 7,3 & 1 & 0 & & & \\
\hline 7 & & $\begin{array}{l}403 \\
414\end{array}$ & 319 & 891 & 572 & $\begin{array}{l}91,8 \\
99,3\end{array}$ & $\begin{array}{l}52,5 \\
53,3\end{array}$ & $\begin{array}{l}39,5 \\
34,5\end{array}$ & & 26,3 & 15,2 & 5,3 & 0,5 & 0 & \multirow{2}{*}{\multicolumn{2}{|c|}{3}} \\
\hline 7 & $\begin{array}{l}3,76 \\
3,15\end{array}$ & $\begin{array}{l}414 \\
382\end{array}$ & $\begin{array}{l}336 \\
303\end{array}$ & $\begin{array}{l}962 \\
904\end{array}$ & $\begin{array}{l}626 \\
601\end{array}$ & $\begin{array}{l}99,3 \\
96\end{array}$ & $\begin{array}{l}53,3 \\
40,9\end{array}$ & $\begin{array}{l}34,3 \\
29,3\end{array}$ & $\begin{array}{l}27,1 \\
23,9\end{array}$ & $\begin{array}{l}22 \\
17.2\end{array}$ & $\begin{array}{l}16,3 \\
11,8\end{array}$ & $\begin{array}{l}8,8 \\
4,3\end{array}$ & $\begin{array}{l}1,4 \\
0\end{array}$ & 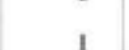 & & \\
\hline 8 & 5,47 & 417 & 281 & 869 & 588 & 84,7 & 52 & 42,3 & 33,2 & 24,5 & 14,1 & $\begin{array}{l}4,3 \\
2\end{array}$ & $\begin{array}{l}0 \\
0\end{array}$ & & \multicolumn{2}{|l|}{7} \\
\hline & 3,72 & 385 & 272 & 868 & 596 & 74 & 43,1 & 35,2 & 27,3 & 21,5 & 12,8 & 1,6 & 0 & 3 & \multirow{2}{*}{\multicolumn{2}{|c|}{24}} \\
\hline 9 & 4,04 & 471 & 375 & 889 & 514 & 100 & 78,3 & 45 & 36,5 & 31 & 21 & 7,9 & 0 & & & \\
\hline & 3,18 & 435 & 354 & 942 & 588 & 99,2 & 63,9 & 40,3 & 30,5 & 26,1 & 19,9 & 11,7 & 0 & 7 & \multirow{2}{*}{\multicolumn{2}{|c|}{26}} \\
\hline 10 & $\begin{array}{r}3,86 \\
2,89\end{array}$ & 445 & 358 & 869 & 511 & 100 & 69,8 & 45,1 & 35,6 & 27 & 11,4 & 3,1 & 0 & & & \\
\hline 13 & $\begin{array}{l}2,89 \\
4,23\end{array}$ & $\begin{array}{l}412 \\
420\end{array}$ & $\begin{array}{l}327 \\
324\end{array}$ & $\begin{array}{l}907 \\
954\end{array}$ & $\begin{array}{l}580 \\
630\end{array}$ & $\begin{array}{l}99,1 \\
99,4\end{array}$ & $\begin{array}{l}54,7 \\
49,3\end{array}$ & $\begin{array}{l}41,9 \\
37,6\end{array}$ & & 25,5 & 17,2 & 8,8 & 0 & -2 & \multirow{2}{*}{\multicolumn{2}{|c|}{2}} \\
\hline & 3,46 & 398 & 333 & 922 & 589 & $\begin{array}{l}700,4 \\
100\end{array}$ & $\begin{array}{l}49,3 \\
51,6\end{array}$ & $\begin{array}{l}37,6 \\
32,2\end{array}$ & $\begin{array}{l}29,3 \\
24,2\end{array}$ & $\begin{array}{l}23,3 \\
19,9\end{array}$ & $\begin{array}{l}15,8 \\
12,5\end{array}$ & $\begin{array}{l}9 \\
6,3\end{array}$ & $\begin{array}{l}0,8 \\
0,3\end{array}$ & 3 & & \\
\hline 14 & 4,18 & 455 & 386 & 1001 & 615 & 100 & 74,3 & 46,4 & 35,3 & 27,9 & 22,8 & 1,4 & $\begin{array}{l}0,3 \\
0,3\end{array}$ & & \multicolumn{2}{|l|}{4} \\
\hline & 3,47 & 405 & 321 & 921 & 600 & 99,5 & 46,7 & 34,8 & 29,3 & 23,2 & 16,4 & 7,6 & 0 & 3 & \multirow{2}{*}{\multicolumn{2}{|c|}{1}} \\
\hline 15 & 3,30 & 458 & 417 & 1079 & 662 & 100 & 81,3 & 48,2 & 34,2 & 28,3 & 23,3 & 15,8 & 4,9 & & & \\
\hline 17 & $\begin{array}{l}2,53 \\
4,42\end{array}$ & 444 & $\begin{array}{l}368 \\
345\end{array}$ & $\begin{array}{l}958 \\
834\end{array}$ & $\begin{array}{l}590 \\
489\end{array}$ & 100 & $\begin{array}{l}76,2 \\
65,4\end{array}$ & 46,2 & 36,5 & 29,8 & 22,2 & 13,3 & 4 & 2 & \multirow{2}{*}{\multicolumn{2}{|c|}{29}} \\
\hline & $\begin{array}{l}4,42 \\
3,03\end{array}$ & $\begin{array}{l}414 \\
384\end{array}$ & $\begin{array}{l}345 \\
302\end{array}$ & $\begin{array}{l}834 \\
847\end{array}$ & $\begin{array}{l}489 \\
545\end{array}$ & $\begin{array}{l}99,7 \\
94,3\end{array}$ & $\begin{array}{l}65,4 \\
43\end{array}$ & $\begin{array}{l}42,4 \\
31,6\end{array}$ & $\begin{array}{l}31,8 \\
25,2\end{array}$ & $\begin{array}{l}21,7 \\
18,4\end{array}$ & $\begin{array}{l}7,7 \\
8,6\end{array}$ & 1,3 & 0 & & & \\
\hline 18 & 4,62 & 421 & 369 & 800 & 431 & $\begin{array}{l}94,3^{3} \\
100\end{array}$ & 67,6 & $43^{\circ}$ & 28,8 & $\begin{array}{l}18,4 \\
17,9\end{array}$ & $\begin{array}{l}8,6 \\
3,3\end{array}$ & $\begin{array}{l}1,5 \\
0\end{array}$ & $\begin{array}{l}0 \\
0\end{array}$ & 1 & \multirow{2}{*}{\multicolumn{2}{|c|}{32}} \\
\hline & 2,82 & 400 & 363 & 922 & 559 & 98 & 55,1 & 36,2 & 27,5 & 22 & 14,5 & 8,4 & 0 & 5 & & \\
\hline 19 & 5,28 & 409 & 341 & 866 & 525 & 100 & 56,7 & 34,6 & 26,1 & 18,8 & 7,9 & 1,5 & 0 & & \multirow{2}{*}{23} & \\
\hline 20 & $\begin{array}{l}4,33 \\
4,39\end{array}$ & $\begin{array}{l}391 \\
432\end{array}$ & $\begin{array}{l}319 \\
364\end{array}$ & $\begin{array}{l}877 \\
918\end{array}$ & 558 & 97,3 & $\begin{array}{l}39,2 \\
67\end{array}$ & $\begin{array}{l}30,1 \\
40,9\end{array}$ & $\begin{array}{l}23,6 \\
32,2\end{array}$ & 16.5 & 8,5 & 1,4 & 0 & 1 & & 19 \\
\hline & $\begin{array}{l}4,39 \\
2,96\end{array}$ & $\begin{array}{l}432 \\
417\end{array}$ & $\begin{array}{l}364 \\
353\end{array}$ & $\begin{array}{l}918 \\
934\end{array}$ & $\begin{array}{l}554 \\
581\end{array}$ & $\begin{array}{l}100 \\
100\end{array}$ & $\begin{array}{l}67 \\
64,2\end{array}$ & 44,7 & $\begin{array}{l}32,2 \\
33,3\end{array}$ & $\begin{array}{l}25,2 \\
27,6\end{array}$ & $\begin{array}{l}12,9 \\
18\end{array}$ & $\begin{array}{l}6 \\
8,8\end{array}$ & $\begin{array}{l}0,4 \\
2,3\end{array}$ & 1 & 12 & 9 \\
\hline
\end{tabular}




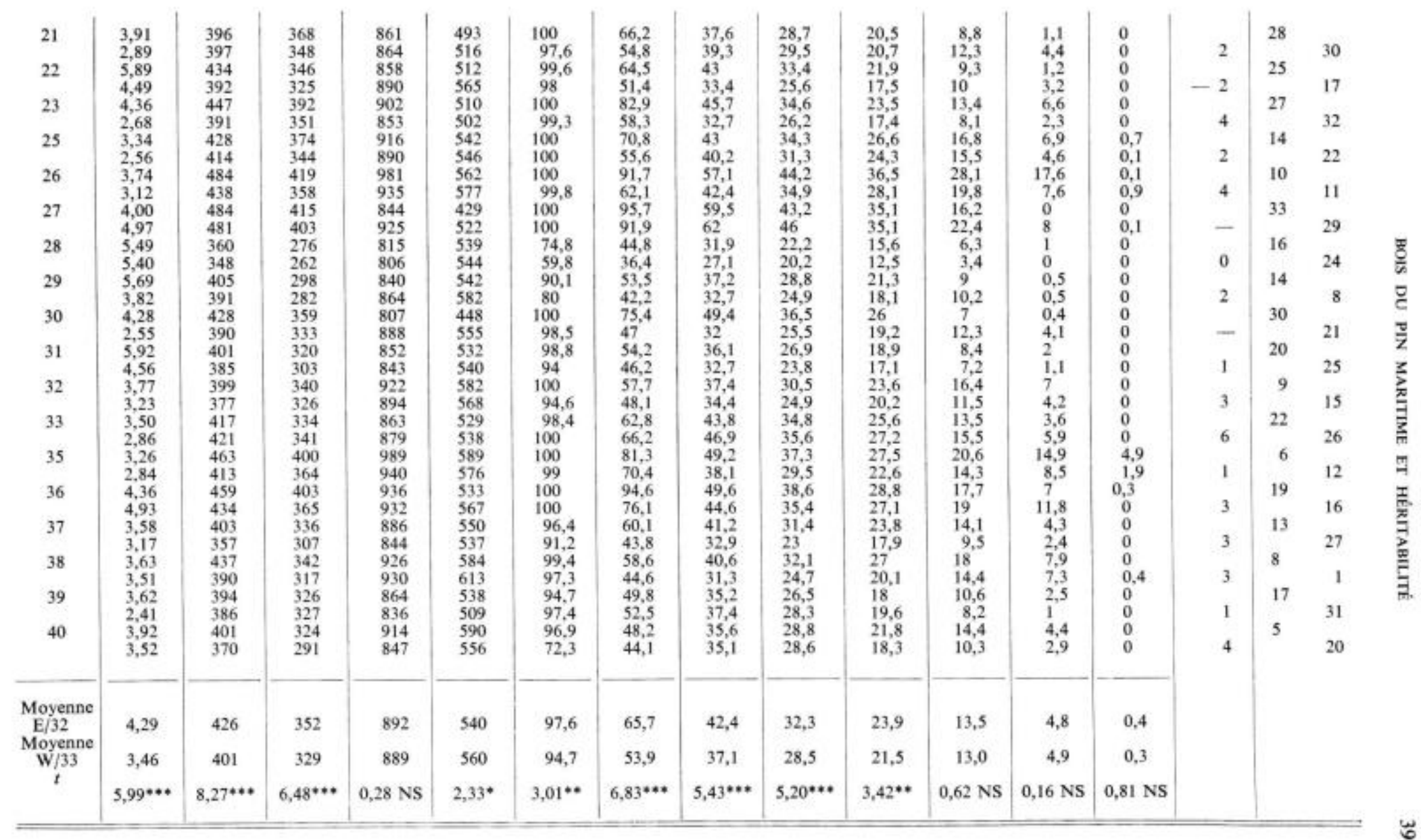



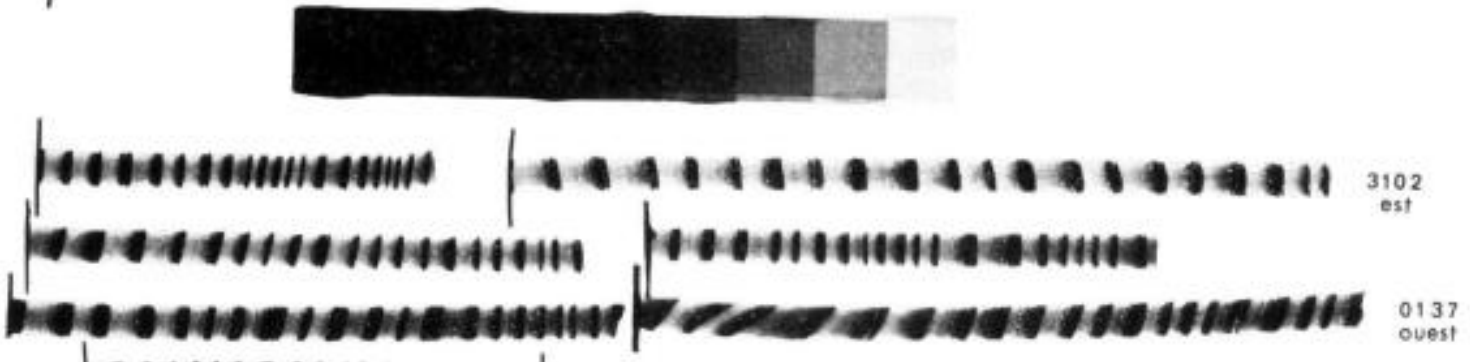

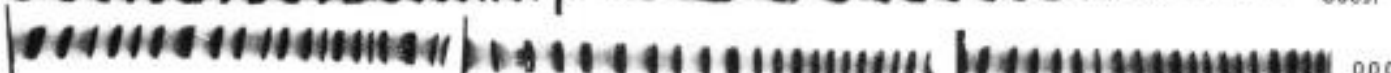

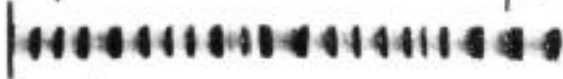

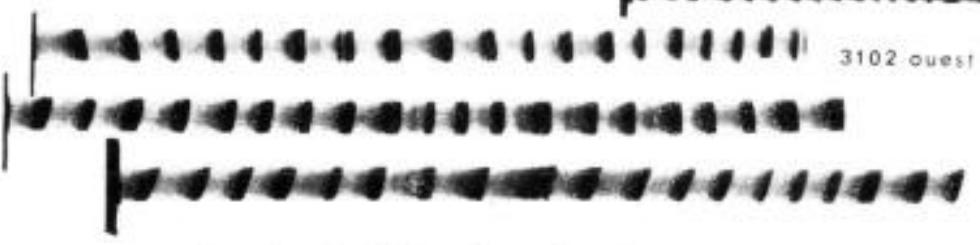

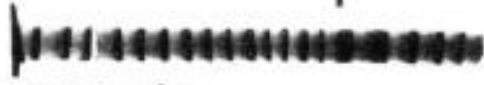

FiG, 1. - Positif de radiographies des carottes de sondage d'arbres-mères Les cernes annuels représentent la période allant de la $1 l^{\circ}$ à la $30^{\circ}$ annie de la vie de cer arbres. 0137 est l'arbre-mère à l'infradensité la plus élevée (475 $\mathrm{g}^{\prime}\left(\mathrm{dm}^{3}\right)$; son hétérogénéité $\mathrm{D}-\mathrm{d}$ est faible

3102 est Tarbre-mére à Finfradensité la plus faible (355 g/dw $\left.{ }^{3}\right)$.

0002 est un arbre à hétérogénéité D-d elevée $\left(626 \mathrm{~g} / \mathrm{dm}^{2}\right)$.

Fig. 1. - Positive prints of radiographies about 20 rings of some mother-trees (from the 1 (th to the 30 th year).

0137: mother-tree with the highest mean specific gravity $\left(475 \mathrm{~g} / \mathrm{dm}^{3}\right)$ and a low heterogeneity D -

$\left(429 \mathrm{~g} / \mathrm{dm}^{2}\right)$

3102 : mother-tree with the lowest mean specific gravity $\left(355 \mathrm{~g} / \mathrm{dm} \mathrm{m}^{3}\right)$.

0002 ; mother-tree with a high heterogeneify $\left(626 \mathrm{~g} / \mathrm{dm}^{3}\right)$. 
analyses factorielles; seuls quelques arbres ont un pourcentage différent de zéro au niveau des $1000 \mathrm{~g} / \mathrm{dm}^{3}$.

L'écart moyen de densité D - d (figure 1) entre les densités maximales et les densités minimales s'élève à $554 \mathrm{~g} / \mathrm{dm}^{3}$. Les arbres qui présentent un écart faible ou fort pour l'un des orientements ne le présentent pas forcément pour l'autre.

L'écart de densité semble jouer un rôle particulier dans les calculs d'analyse factorielle. Il apparait en effet comme variable discriminante dans le cas des orientements est, ouest, et des valeurs relatives aux moyennes de ces deux orientements.

Pour le côté est, D - d intervient concurremment avec les pourcentages aux niveaux $500 \mathrm{~g} / \mathrm{dm}^{3}$ ou $600 \mathrm{~g} / \mathrm{dm}^{3}$. L'hétérogénéité est liée à la variable synthétique par un coefficient de corrélation $r=0,89$; pour le côté ouest, on trouve entre $\mathrm{D}-\mathrm{d}$ et la variable synthétique un coefficient $r=0,94$. Dans le cas des valeurs moyennes de l'est et de l'ouest, ce coefficient est : $r=0,95$.

\subsection{Largeur des accroissements annuels, rendement en fibres, longueur de fibres.}

Entre 11 et 30 ans, les accroissements annuels moyens valent $3,87 \mathrm{~mm}: 4,38 \mathrm{~mm}$ (E) et $3,44 \mathrm{~mm}(\mathrm{~W})$.

Cette caractéristique de croissance n'est jamais fortement liée aux variables synthétiques des analyses factorielles faites sur les deux orientements et leur moyenne; elle ne donne pas lieu à des coefficients de corrélation élevés et ne sert pas dans la différenciation des arbres.

Sur chaque arbre-mère, on a mesuré à partir des carottes sondées en oblique le rendement en fibres, ainsi que la longueur des fibres; du côté du cœur, le rendement en fibres atteint $49,0 \%: 49,4 \%$ (E) et $48,8 \%(\mathrm{~W})$; du côté de l'écorce, on a trouvé $49,7 \%: 48,7 \%(\mathrm{E})$ et $50,7 \%(\mathrm{~W})$.

Les longueurs de fibres sont, pour la zone du cour $3,50 \mathrm{~mm}: 3,39$ (E) et 3,61 (W) et pour la zone proche de l'écorce $3,97 \mathrm{~mm}: 3,82$ (E) et 4,13 (W).

Pour l'analyse factorielle relative au côté est, la longueur des fibres dans la zone du cơur apparait comme étant liée à une variable synthétique avec un coefficient de corrélation $r=0,79$ un peu supérieur à celui qui lie, dans le même calcul, cette variable au pourcentage de bois dont la densité dépasse $300 \mathrm{~g} / \mathrm{dm}^{3}(r=0,74)$. Une observation analogue peut être faite au sujet du côté ouest pour lequel on a trouvé, dans les mêmes conditions, un coefficient $r=0,90$, ainsi que pour les valeurs moyennes des côtés est et ouest pour lesquelles on a $r=0,85$.

\subsection{Angle de la fibre torse.}

Cet angle, formé par la direction générale des fibres avec l'axe de l'arbre et apprécié sur la couche de bois la plus externe, tend à se stabiliser avec l'âge et prend le plus souvent des valeurs positives. Il est donné ici à titre indicatif, l'âge exact des arbres-mères n'étant pas connu.

L'angle a été mesuré du côté ouest à $4 \mathrm{~m}$ de hauteur pour éviter les zones de cicatrisation des cares de gemmage. Le tableau 3 donne la valeur de ces angles pour 31 arbres-mères. Seuls deux d'entre eux ont un pas à gauche avec un angle de -2 grades. Les arbres à fibre torse la 
plus marquée présentent des valeurs de 6 à $7 \mathrm{gr}$ avec un pas à droite. Trois arbres en sont à la phase d'annulation de leur angle, qui correspond probablement au passage d'un pas négatif à un pas positif.

Comme on le verra à propos des demi-frères du test D 441, descendants de ces arbresmères, il ne faut pas attacher une valeur définitive à ces angles qui évoluent pendant une partie relativement longue de la vie de ces plantes, avant de se stabiliser.

\subsection{Anisotropie relative aux 20 cernes allant de 11 à 30 ans.}

L'anisotropie se manifeste par des différences parfois sensibles entre les valeurs des caractéristiques mesurées à l'est et à l'ouest des arbres. On considère que ces différences sont dues à la présence presque constante de bois anormal de compression dans la partie est des troncs des pins maritimes croissant dans le massif landais (H. PoLge et G. ILLY, 1967).

Ce phénomène est plus ou moins marqué selon les caractéristiques envisagées. Il est très net en ce qui concerne la largeur des accroissements annuels : les cernes du côté est sont plus larges que les cernes ouest de $24,6 \%$. Le test de comparaison de moyennes donne $t=5,99$ qui est significatif à $1 \%$. Il est très marqué également pour la densité globale : $t$ valant 8,27 . L'anisotropie apparaît bien aussi dans le cas des divers pourcentages $1 d<300 \mathrm{~g} / \mathrm{dm}^{3}$ à $1 d<700 \mathrm{~g} / \mathrm{dm}^{3}$. Le test de comparaison de moyennes n'est plus significatif qu'au seuil de $5 \%$ lorsqu'on considère l'hétérogénéité $\mathrm{D}-d$; pour cette grandeur, c'est d'ailleurs le côté ouest qui est le plus contrasté ( $560 \mathrm{~g} / \mathrm{dm}^{3} \mathrm{~d}$ 'écart contre $540 \mathrm{~g} / \mathrm{dm}^{3}$ pour le côté est).

Enfin, on n'a pu mettre en évidence de différences entre les côtés est et ouest que lorsqu'on a considéré les pourcentages à 800,900 et $1000 \mathrm{~g} / \mathrm{dm}^{3}$ et la densité maximum. L'anisotropie semble ici être le fait de la largeur des cernes, de la densité minimum et des niveaux de densité inférieurs ou égaux à $700 \mathrm{~g} / \mathrm{dm}^{3}$; les hauts niveaux et la densité maximale sont identiques dans les deux côtés des arbres et ne paraissent pas être responsables des écarts génẻralement observés.

Bien que des calculs de comparaison de moyenne n'aient pas été faits pour les longueurs de fibres, on peut toutefois noter que, tant du côtẻ du cœur que de celui de l'écorce, le côté est a des fibres plus courtes que le côté ouest, d'environ 6 à $7 \%(3,61 \mathrm{~mm}$ (E) et $3,87 \mathrm{~mm}$ (W)). Les résultats relatifs aux rendements en fibres sont tantôt en faveur de l'est, tantôt en faveur de l'ouest et, avec le degré de précision que l'on a, il est donc impossible d'affirmer qu'il y a, pour cette variable, une hétérogénéité.

\section{2. - Test D 44I}

\subsection{Densité globale.}

La densité globale a été mesurée chez les descendants du test D 441 à la fois sur les échantillons provenant des sondages droits et sur ceux des sondages obliques. Les résultats relatifs auxs ondages droits sont un peu différents des résultats relatifs aux sondages obliques, car tout arbre ayant subi le sondage du premier type est demi-frère de l'arbre voisin ayant subi le sondage du deuxième type (les deux types de sondages n'étaient pas faits sur le même individu). 
TABLEAU 4 - TABLE 4

Mesures sur les familles du test D 441. Measserements of the families of the test D 441.

\begin{tabular}{|c|c|c|c|c|c|c|c|c|c|}
\hline \multirow[t]{2}{*}{ Code } & \multicolumn{2}{|c|}{ Densitê } & \multicolumn{2}{|c|}{$\begin{array}{l}\text { Pourcentage } \\
\text { de résine }\end{array}$} & \multicolumn{2}{|c|}{$\begin{array}{l}\text { Longueur } \\
\text { des fibres }\end{array}$} & \multicolumn{2}{|c|}{$\begin{array}{l}\text { Rendement } \\
\text { en fibres }\end{array}$} & \multirow{2}{*}{$\begin{array}{c}\begin{array}{c}\text { Angle de } \\
\text { la fibre } \\
\text { torse }\end{array} \\
\text { W }\end{array}$} \\
\hline & $\mathrm{E}$ & w & $\mathrm{E}$ & w & $E$ & w & E & w & \\
\hline 1 & 329,1 & 316,6 & 12,9 & 12,8 & 1,568 & 1,641 & 46,4 & 47,2 & $-7,7$ \\
\hline 2 & 310,1 & 302,6 & 9,9 & 12,6 & 1,689 & 1,660 & 45,6 & 45,4 & -6.9 \\
\hline 3 & 308,1 & 292,9 & 11.4 & 12,0 & 1,649 & 1,726 & 44,7 & 46,0 & $\begin{array}{r}-5,0 \\
\end{array}$ \\
\hline 4 & 316,7 & 309,9 & 11,4 & 13,0 & 1,631 & 1,719 & 48,3 & 46,5 & -4.7 \\
\hline 5 & 319,1 & 305,6 & 11.2 & 11,6 & 1,606 & 1,685 & 45,9 & 47,5 & $-6,2$ \\
\hline 6 & 311,3 & 304,9 & 12,3 & 12,6 & 1,592 & 1,622 & 45.3 & 46,7 & $-4,7$ \\
\hline 7 & 326,7 & 319,1 & 10,0 & 12,3 & 1,687 & 1.723 & 46,7 & 45,7 & $-6,1$ \\
\hline 8 & 302,9 & 296,0 & 12,7 & 12,9 & 1,621 & 1,734 & 42,0 & 49,1 & $-4,1$ \\
\hline 9 & 336,3 & 322,7 & 12,2 & 12,5 & 1,609 & 1.613 & 46,9 & 46,9 & $-6,0$ \\
\hline 10 & 308,4 & 302,9 & 10,4 & 13.8 & 1,614 & 1,734 & 46,1 & 47,3 & $-4,9$ \\
\hline 11 & 337,1 & 319,9 & 10,9 & 13,1 & 1,619 & 1,763 & 46,0 & 46,5 & $-4,6$ \\
\hline 12 & 315.4 & 314,7 & 10,2 & 12,1 & 1,608 & 1,671 & 44,8 & 46,9 & $-5,5$ \\
\hline 13 & 304,1 & 296,3 & 13,2 & 13,3 & 1,561 & 1,643 & 41,5 & 45,0 & $-5,1$ \\
\hline 14 & 325,0 & 319,0 & 10.7 & 12,6 & 1,574 & 1,684 & 45,5 & 45.5 & $-6,0$ \\
\hline 15 & 307,7 & 305,9 & 11,7 & 12,7 & 1.660 & 1,758 & 45,6 & 47,0 & -4.9 \\
\hline 16 & 321,4 & 312,1 & 10.0 & 11,6 & 1,601 & 1.697 & 45,3 & 47.1 & $-3,6$ \\
\hline 17 & 315,1 & 310,1 & 11,1 & 13,4 & 1.626 & 1,639 & 43,8 & 47.3 & -5.6 \\
\hline 18 & 325,6 & 312,9 & 11.9 & 11,7 & 1.590 & 1,699 & 44,1 & 47,7 & $-5,8$ \\
\hline 19 & 310,3 & 307.3 & 12,8 & 13,4 & 1.542 & 1,685 & 45,8 & 50,0 & -6.6 \\
\hline 20 & 323,1 & 308,4 & 10.9 & 13,2 & 1.622 & 1,690 & 43,0 & 46,6 & -5.8 \\
\hline 21 & 311,8 & 298,1 & 12.1 & 13,5 & 1,667 & 1,674 & 46,3 & 45,2 & $-6,0$ \\
\hline 22 & 317,6 & 302,3 & 12,1 & 12,9 & 1,620 & 1,705 & 44.7 & 44,9 & $-4,9$ \\
\hline 23 & 315,6 & 311,9 & 11.1 & 12,0 & 1,639 & 1,621 & 44,9 & 43,7 & $-6,1$ \\
\hline 24 & 317,9 & 302,7 & 12,0 & 13,0 & 1,640 & 1,653 & 44,3 & 44,2 & $-5,0$ \\
\hline 25 & 326,3 & 320,4 & 12,7 & 13,0 & 1,633 & 1,677 & 43,7 & 48,0 & $-4,1$ \\
\hline 26 & 321,9 & 313,4 & 10,7 & 11.5 & 1,595 & 1,672 & 46,2 & 44,2 & $-6,7$ \\
\hline 27 & 315,6 & 309,7 & 10,0 & 11.4 & 1,642 & 1,660 & 44,0 & 48,4 & $-4,5$ \\
\hline 28 & 310,9 & 305,7 & 11.8 & 12,1 & 1,624 & 1.716 & 45,8 & 48,4 & $-6,3$ \\
\hline 29 & 319,3 & 311,0 & 12.6 & 12,9 & 1,649 & 1.779 & 43,2 & 45,1 & $-5,1$ \\
\hline 30 & 313,3 & 314,3 & 10,4 & 11,9 & 1,683 & 1,738 & 44.7 & 44,9 & $-5,4$ \\
\hline 31 & 317,7 & 307,4 & 12,9 & 11,5 & 1,604 & 1,645 & 43,3 & 45,3 & $-4,0$ \\
\hline 32 & 308,6 & 302,1 & 10,8 & 12,0 & 1.656 & 1.676 & 48,7 & 49,0 & $-5,2$ \\
\hline 33 & 314,9 & 310,1 & 11,5 & 12,2 & 1,603 & 1,664 & 43.4 & 45,6 & $-6,1$ \\
\hline 34 & 304,3 & 294,6 & 10,0 & 11,5 & 1,634 & 1,701 & 44.8 & 43,3 & $-4,4$ \\
\hline 35 & 329,1 & 328,3 & 10,7 & 12,0 & 1.625 & 1,641 & 47,3 & 46,5 & $-6,2$ \\
\hline 36 & 328,0 & 325,1 & 10,5 & 12,3 & 1,571 & 1,640 & 47,8 & 45,6 & $-7,5$ \\
\hline 37 & 320,1 & 305,3 & 10,6 & 11.8 & 1,629 & 1,741 & 47,4 & 46,4 & $-4,4$ \\
\hline 38 & 320,4 & 308,7 & 11,9 & 12,4 & 1,559 & 1,650 & 44,9 & 46.1 & $-6,8$ \\
\hline 39 & 330.9 & 308,3 & 10,5 & 12,6 & 1,603 & 1,721 & 42,3 & 48,9 & $-5,5$ \\
\hline 40 & 316,1 & 301,3 & 12,0 & 13.0 & 1,622 & 1,665 & 45,9 & 46,8 & $-5,4$ \\
\hline 41 & 324,4 & 317,3 & 12,6 & 13,0 & 1,593 & 1,660 & 44,2 & 46,0 & $-5,5$ \\
\hline \multirow[t]{2}{*}{ Moyenne } & 318,0 & 309,2 & 11,4 & 12,5 & 1,619 & 1,685 & 45,1 & 46,5 & $-5,5$ \\
\hline & \multicolumn{2}{|c|}{$t-11,35 * \cdots$} & \multicolumn{2}{|c|}{$t=7,54^{\cdots \cdots}$} & \multicolumn{2}{|c|}{$t=10,51 * \cdots$} & \multicolumn{2}{|c|}{$t=3,82 \cdots$} & \\
\hline
\end{tabular}



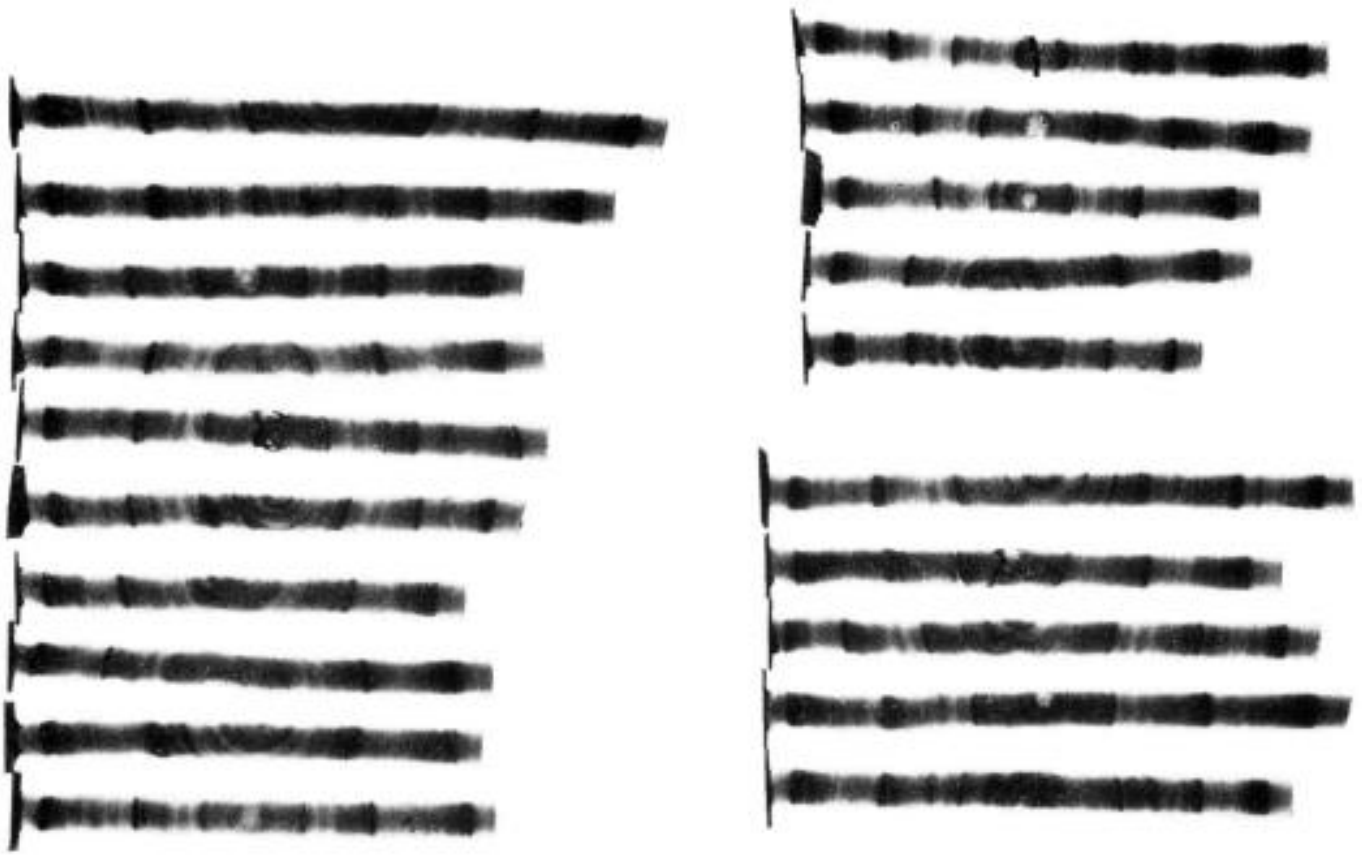

F1G. 2. - Positifs de radiographies montrant:

- à gauche : les 10 carottes de soniage est-owest de la famille 0101 a faible infradensité ( $\left.301 \mathrm{~g} / \mathrm{dm}^{\mathrm{2}}\right)$.

- à droite : les 10 carottes de sondage est-ouest de la famille 0013 a forte infradensite $\left(3 / 8 \mathrm{~g} / \mathrm{dm}^{3}\right.$ ).

Le cốté est se trouve à droite des photos.

F16. 2. - Positive prints of radiographies showing :

- on the left hand : 10 increment borings (east-west) taken from the family olot with a low mean specific gravity $\left(301 \mathrm{~g} / \mathrm{dm}^{3}\right)$.

- on the right hand: 10 increment borings (cast-west) taken from the family 0013 with a high mean specific gravity $\left(3 / 8 \mathrm{~g} / \mathrm{dm}^{3}\right)$.

The east side is on the right hand.

Diverses déterminations de la densité ont été faites côtés est et ouest séparés, carottes droites et obliques séparées, ou au contraire, côtés et modalités confondus. Les principaux résultats sont reportés dans le tableau 4.

Si l'on regroupe les prélèvements droits et obliques, ainsi que les côtés est et ouest, on trouve que la densité de ces arbres jeunes varie de $298 \mathrm{~g} / \mathrm{dm}^{3}$ (descendance 5303 ) à $331 \mathrm{~g} / \mathrm{dm}^{3}$ (descendance 0011 ) et présente donc un intervalle de variation de $33 \mathrm{~g} / \mathrm{dm}^{3}$ (figure 2 ).

\subsection{Composantes de la densité.}

Elles ont été évaluées sur ces arbres très jeunes à partir des mesures faites sur les deux saisons de végétation 1965 et 1966; rappelons à ce sujet que les arbres n'étaient alors ãgés que de 5 ans et demi lors des prélèvements faits en juin 1967 et que, par conséquent, ils se trouvaient dans leur phase juvénile.

On a calculé trois pourcentages définissant, d'une manière comparable à ce qui a été fait chez les arbres-mères, les proportions de bois de densité supérieure à $350 \mathrm{~g} / \mathrm{dm}^{3}, 400 \mathrm{~g} / \mathrm{dm}^{3} \mathrm{et}$ 
$450 \mathrm{~g} / \mathrm{dm}^{3}$. Les densités minimales annuelles et maximales annuelles, ainsi que la largeur des cernes ont également été mesurées, la différence entre les deux premières chiffrant l'hétérogénéité de la matière ligneuse (tableau 5).

A cette phase de leur vie, les arbres produisent un bois de densité assez faible, aussi n'estil pas surprenant de constater qu'il n'y a sur le rayon qu 'une proportion de $21,3 \%$ de ce bois qui dépasse la densité de $350 \mathrm{~g} / \mathrm{dm}^{3}$. Pour les niveaux supérieurs, ces pourcentages décroissent fortement; on ne trouve plus que $11,6 \%$ de bois de densité supérieure à $400 \mathrm{~g} / \mathrm{dm}^{3} \mathrm{et}$ $5,0 \%$ dépassent le niveau $450 \mathrm{~g} / \mathrm{dm}^{3}: 5,6 \%$ (E) et $4,5 \%$ (W). La plus grande partie de la matière ligneuse (soit $79 \%$ sur la largeur d'un cerne) est donc constituée de bois dont la densité est comprise entre $245 \mathrm{~g} / \mathrm{dm}^{3} \mathrm{ct} 350 \mathrm{~g} / \mathrm{dm}^{3}$.

La valeur moyenne des minima est $245 \mathrm{~g} / \mathrm{dm}^{3}$ et celle des maxima $477 \mathrm{~g} / \mathrm{dm}^{3}$, ce qui conduit à $232 \mathrm{~g} / \mathrm{dm}^{3}$ pour l'estimation de l'hétérogénéité moyenne (figure 3 ).
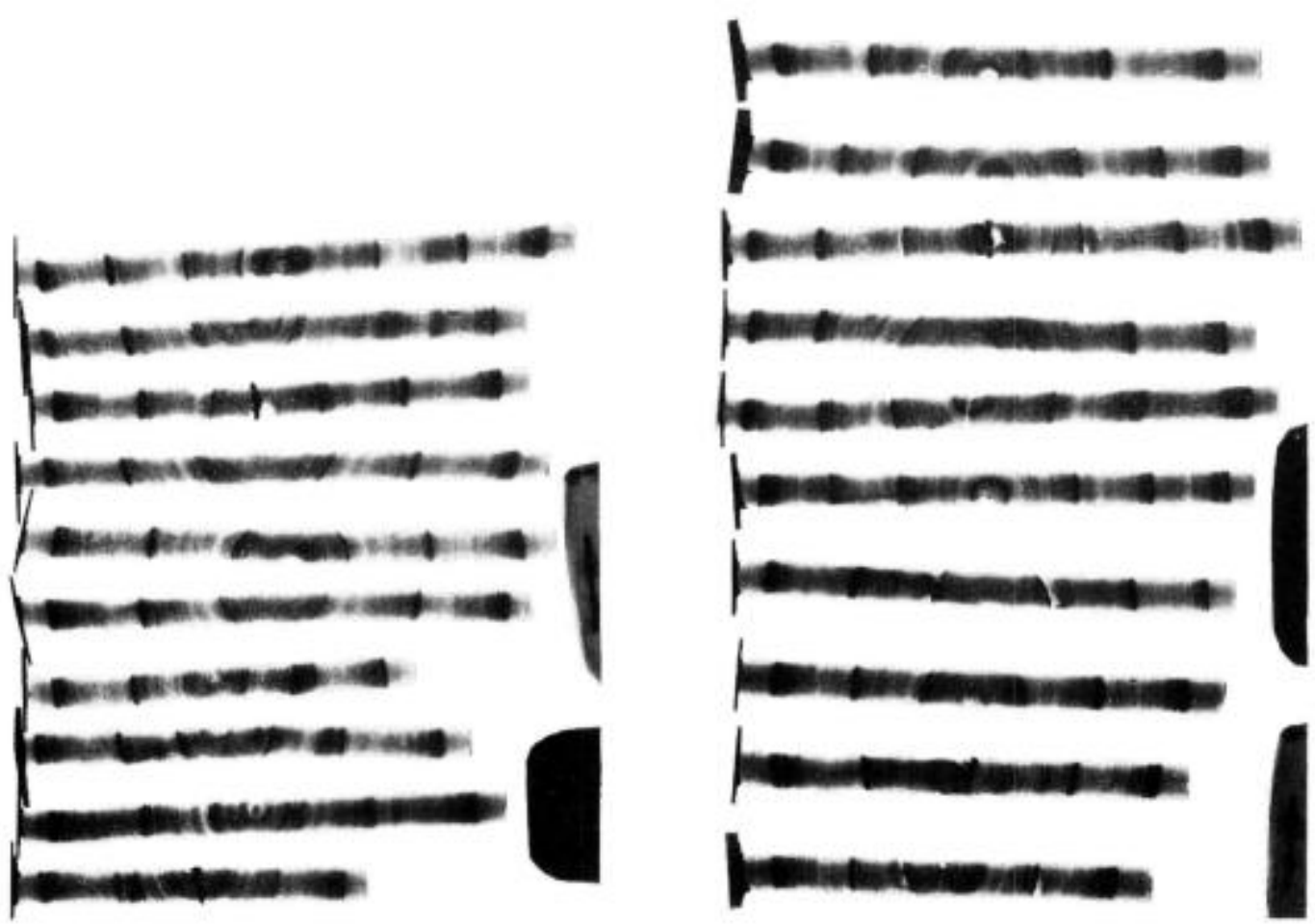

FIG. 3. - Demi-frères du test D 441 - Positifs de radiographies montrant :

- à gauche : les 10 carottes de sondage est-ouest de la famille 5308 à hétérogénéité D - d faible (2006 g/dm²) et à infradensite $\left(3 / 2 \mathrm{~g} / \mathrm{dm}^{3}\right)$ voisine de la moyenne.

- à droite : les 10 caroltes de sondage ext-ouest de la famille 5302 à hétérogénéité D - d forte $\left(272 \mathrm{~g}^{\prime} / \mathrm{m}^{\mathrm{2}}\right)$ et d̀ infradensité ( $\left.315 \mathrm{~g} / \mathrm{dm}^{3}\right)$ voisine de la moyenne.

Le côté est se trouve à droite des photos.

Fig. 3. - Half sibs of the D 441 progeny test - Positive prints of radiographies showing

on the left hand: 10 increment borings (east-west) taken from the family 5308 with a low heterogeneity ( $\left.206 \mathrm{~g} / d \mathrm{~m}^{3}\right)$ and with a mean specific gravity neither too high nor too low.

- on the right hand : 10 increment borings (east-west) taken from the family 5302 with a high heterogeneily $\left(562 \mathrm{~g} / \mathrm{dm}^{3}\right)$ and with a mean specific gravity neither too high nor too low.

The east side is on the right hand. 
Cette dernière caractéristique est d'ailleurs en pleine évolution à ce stade de la vie de l'arbre, et elle tend à augmenter régulièrement d'année en année, les valeurs des densités maximales croissant elles-mêmes plus rapidement que celles des densités minimales. L'hétérogénéité de l'année 1965 vaut $229 \mathrm{~g} / \mathrm{dm}^{3}$; elle est passée à $238 \mathrm{~g} / \mathrm{dm}^{3}$ en 1966 . La fin de cette tendance n'apparait qu'avec la formation du bois adulte qui, elle, semble plus dépendante des diverses conditions de milieu, le climat en particulier.

Cette caractéristique importante de la qualité du bois a fait l'objet de calculs d'héritabilité comme on le verra ci-après au chapitre 3 .

\subsection{Largeur des accroissements annuels, rendement en fibres, longueur de fibres.}

Les accroissements annuels de ces jeunes arbres sont importants : pour $1965: 4,6 \mathrm{~mm}$ sur le rayon en moyenne, et pour 1966:4,3 mm. La moyenne générale des deux saisons de végétation et des deux orientements vaut $4,47 \mathrm{~mm}$. Des différences significatives existent entre les diverses familles, mais les tendances observées, sans pourtant être bouleversées lorsqu'on compare les résultats de deux années successives, ne sont pas forcément stables, ne serait-ce que sur un intervalle de temps de 5 années. Mềme si, pour une caractéristique $\mathrm{X}$, le coefficient de corrélation entre l'état ả l'année $n$, soit $\mathrm{X}_{\mathrm{n}}$, et l'état de l'année $n+1$, soit $\mathrm{X}_{\mathrm{n}}+{ }_{1}$, revêt des valeurs significatives élevées (voir le tableau 9 en annexe), il n'est pas certain que les coefficients calculés sur les valeurs $X_{n}$ et $X_{n}+{ }_{5}$ ou $X_{n}+{ }_{10}$ soient significatifs. Les classements établis à l'année $n$ subsistent donc souvent, à quelques modifications près, à l'année $n+1$, mais sont susceptibles de se remanier au point que l'état à $n+5$ ou $n+10$ soit tout différent, une succession de corrélations même élevées n’étant pas équivalente à des translations d'états identiques stables.

L'analyse de variance faite sur les rendements en fibres ne fait pas apparaitre de différences significatives entre les diverses familles; le rendement moyen est de $45,8 \%$.

Dans le cas des longueurs de fibres, on a trouvé une différence conduisant à un test $\mathrm{F}$ légèrement inférieur à la limite de signification $\left(\mathrm{F}_{258}^{10}-1,38\right)$ pour le côté ouest des arbres; pour ce qui est du côté est, on n'a pu mettre en évidence une telle différence entre les familles. A cet âge de 5,5 ans, la longueur moyenne des fibres atteint $1,65 \mathrm{~mm}$.

\subsection{Teneur en résine.}

Cette caractéristique semble actuellement retenir moins l'attention que d'autres propriétés du bois, au point qu'il n'y est même pas fait allusion dans des études récentes (Nicholls, Perry, Hopkins, 1968 op, cit.) qui prennent seulement en considération la fibre torse, la longueur des trachéides, la densité du bois, le pourcentage de bois d'été et la vitesse de croissance.

Certains indices laissent pourtant penser que cette tendance s'inverse et qu'un regain d'intérêt se manifeste vis-à-vis de la production de la résine. Pour cette raison, et également pour des raisons techniques, nous avons procédé à son extraction des échantillons, et évalué les teneurs correspondantes; un certain nombre de difficultés d'ordre expérimental a fait qu'une partie des résultats est entachée d'erreurs et que quelques-uns seulement peuvent être considérés comme corrects (voir ci-dessous le chapitre 31 et le tableau 6). En moyenne, les prélèvements contenaient $12,0 \%$ de résine. 
Les seules différences de teneur apparues entre familles sont relatives aux côtés est des arbres oủ se forme le bois de réaction; les résultats des côtés ouest n'ont pas permis de les mettre en évidence alors que, chez cette essence, il s'agit là généralement du bois normal. Les valeurs absolues de ces pourcentages semblent un peu élevées; cependant, la concordance observée sur les résultats, obtenus indépendamment dans le cas des carottes droites et dans celui des obliques, autorise à retenir pour l'instant les chiffres donnés au tableau 4 .

\subsection{Angle de la fibre torse.}

L’angle formé par les fibres avec l'axe de l'arbre a été mesuré sur le côté ouest, avant les prélèvements des carottes obliques; aucune différence significative n'a pu être décelée entre les diverses familles. A ce stade de leur existence, tous les arbres ont leurs fibres orientées suivant des hélices tournant à gauche.

En moyenne, cet angle, comptê négativement lorsque le pas est à gauche, vaut - 5,5 grades; il couvre, suivant les descendances, un intervalle allant de $-7,7 \mathrm{gr}$ à $-3,6 \mathrm{gr}$. Ces valeurs n'ont en soi qu'un intérêt limité puisque cet angle varie avec l'âge. Selon des observations personnelles annexes faites sur d'autres pins maritimes, l'angle au début de la vie est voísin de $0 \mathrm{gr}$, soit légèrement supérieur, soit lẻgèrement inférieur. II diminue ensuite rapidement pour atteindre un minimum entre la $15^{\circ} \mathrm{et} \mathrm{la} 30^{\mathrm{L}}$ année; l'angle est alors compris entre - 10 et $-12 \mathrm{gr}$. Par la suite, il augmente plus ou moins rapidement de sorte que, chez certains arbres, dès la $45^{\mathrm{E}}$ année, le sens du pas peut s'inverser, l'angle ayant pris la valeur $0 \mathrm{gr}$ pour tendre asymptotiquement, semble-t-il, vers des valeurs de $+4 \dot{a}+6 \mathrm{gr}$. Ce phénomène d'inversion de l'hélice peut n'apparaitre que bien plus tardivement, après la $70^{\circ}$ année; il est done impossible de juger la fibre torse à partir d'une seule mesure faite sur du bois juvénile en pleine transformation; il faut pouvoir aussi estimer la vitesse de l'évolution de cette caractéristique, ainsi que la date de sa stabilisation en liaison avec l'âge de l'arbre.

Il est apparu aussi que l'angle de la fibre avec l'axe de l'arbre n'est pas constant sur toute une circonférence et qu'il existe donc pour cette caractéristique des variations liées à l'orientement. Sa mesure, en tout cas, semble être délicate et sa signification dépend de la spécification des conditions dans lesquelles elle est faite; des observations analogues ont d'ailleurs été faites sur d'autres essences (KREMPL, 1970).

\subsection{Anisotropie.}

Ce phénomène existe dès les plus jeunes années des arbres (Polge et IlLy, 1967, op. cit.) sur un grand nombre de caractères dont il est parlé dans les paragraphes précédents. Le tableau 5 permet de se rendre compte, pour les 41 familles étudiées, des différences relevées entre les côtés est et ouest (bien que l'anisotropie puisse se manifester suivant d'autres orientements, mais d'une façon moins fréquente).

Des calculs de comparaison de moyennes par séries appariées ont été faits sur les couples de valeurs est et ouest; un test $t$ chiffre le degré de signification des inégalités des caractères.

Les largeurs de cernes sont très significativement plus grandes à l'est qu'à l'ouest, et ceci au seuil de probabilité de $1 \%$ oo. Cette supériorité atteint $4,5 \%$ environ pour 1965 et 1966 , et bien que, en valeur relative, elle soit assez faible, le seuil est élevé du fait que l'inégalité est 
presque toujours au désavantage du côté ouest. Les exceptions sont rares, mais néanmoins existent.

L'anisotropie a pu être décelée également dans le cas de la densité globale du bois (voir tableau $\mathrm{n}^{\circ} 4$ ), le test $t$ atteignant une valeur de 11,36 qui est très significative et montrant que, malgré une différence qui n'atteint que $9 \mathrm{~g} / \mathrm{dm}^{3}$, par rapport aux $309 \mathrm{~g} / \mathrm{dm}^{3} \mathrm{du}$ côté ouest, soit $2,9 \%$, le bois juvénile présente déjà une densité systématiquement plus forte du côté comprimé.

En fait, les renseignements fournis par la densité globale ne décrivent pas complètement le phẻnomẻne. Il se trouve en effet qu'il y a, au sein des caractéristiques de la densité, un gradient d'anisotropie. Celle-ci se manifeste très nettement, avec un seuil de probabilité de $1 \%$ oo, chez les densités minimales de 1965 et 1966 qui sont plus élevées pour le côté est, de $7,3 \%$ et $3,4 \%$ respectivement.

L'anisotropie continue d'apparaittre aux niveaux de densités $350 \mathrm{~g} / \mathrm{dm}^{3}$ et $400 \mathrm{~g} / \mathrm{dm}^{3}$, avec un seuil de signification de $1 \%$ oo; pour le niveau des $400 \mathrm{~g} / \mathrm{dm}^{3}$, le seuil de signification est de $1 \%$. Le phénomène a toujours été mieux marqué en 1966 qu'en 1965. Il tend à disparaître dans les hauts niveaux de densité : en effet, s'il existe encore en 1965 une différence entre les côtés est et ouest dans le cas de la densité maximale, au seuil de signification de $5 \%$, $t$ valant 2,54 pour 40 degrés de liberté, on n'a pu mettre en évidence d'inégalité entre les deux orientements pour l'année 1966 où l'on a trouvé comme densité maximale moyenne $486,5 \mathrm{~g} / \mathrm{dm}^{3}\left(E=487 \mathrm{~g} / \mathrm{dm}^{3} ; \mathrm{W}=486 \mathrm{~g} / \mathrm{dm}^{3}\right)$, la comparaison des différences de densités par rapport à zéro conduisant à une valeur de $t$ non significative de 0,61 .

Ces observations sont de même nature que celles qui ont pu être faites chez les arbresmẻres oủ l'on a vu que l'anisotropie relative aux diverses composantes de la densité se manifeste surtout, outre la densité globale, dans les niveaux allant de $300 \mathrm{~g} / \mathrm{dm}^{3}$ à $700 \mathrm{~g} / \mathrm{dm}^{3}$. alors qu'elle disparait dans les niveaux $800,900,1000 \mathrm{~g} / \mathrm{dm}^{3}$, ainsi que dans le cas des densités maximales. Il semblerait donc que chez le pin maritime l'anisotropie en densité soit surtout le fait du bois de printemps et d'une partie du bois d'été, mais que vers la fin de la saison de végétation, les inégalités de densités disparaissent.

L'hétérogénéité, mesurée par la différence entre la densité maximale et la densité minimale, est anisotrope chez les descendants du test D 441 (de façon moins marquée en 1966 qu'en 1965) et aussi, on l'a vu, chez les arbres-mères, tout au moins sur les 20 cernes allant de 11 à 30 ans étudiés de ce point de vue, mais ceci résulte probablement uniquement de la présence de la densité minimale dans l'expression de cette caractéristique.

Comme on le sait, la teneur en résine des diverses zones des troncs n'est pas constante. Chez les descendants, les extractions faites séparément sur les carottes est et ouest font ressortir une légère supériorité du taux de cette substance dans le côté ouest des arbres $(12,5 \%)$ par rapport au côté est $(11,4 \%)$ qui est confirmée par une valeur élevée du test $t: 7,54$ pour 40 degrés de liberté.

Deux autres caractéristiques sont sujettes à l'anisotropie : ce sont les longueurs des fibres et le rendement en fibres (voir tableau 4). La première des deux se distingue par une différence très significative (au seuil de $1 \%$ oo) entre la longueur moyenne relevéc du côté est, soit $1,619 \mathrm{~mm}$, et celle du côté ouest, soit $1,685 \mathrm{~mm}$, ce qui équivaut à une supériorité de ce dernier orientement d'environ $4 \%$ sur le côté est.

Rappelons à ce sujet que, chez les arbres-mères, on a trouvé un écart comparable et de même sens entre les deux orientements, s'élevant environ à $6,5 \%$. 
La seconde caractéristique relative aux qualités papetières est le rendement en fibres, qui se trouve être inférieur, dans le cas des échantillons provenant du côté est, de $3 \%$ à celui que l'on mesure sur le côté ouest.

II ne semble pas que ces derniers résultats, qui chez les descendants sont significatifs au seuil de $1 \%$ oo, se retrouvent chez les arbres-mères; chez ceux-ci, l'anisotropie du rendement en fibres n'est sensible que dans la zone voisine de l'écorce (l'est a un rendement de $4 \%$ inférieur à l'ouest) et dans la zone du caur, qui correspond au bois jeune, elle est de sens inverse, l'est y ayant un rendement supérieur de $1,2 \%$ à celui de 1 'ouest. Cette dernière constatation n'a d'ailleurs pas de signification au sens statistique. Les résultats relatifs aux descendants sont probablement plus dignes de confiance.

Quelques calculs d'analyse de variance portant sur les 41 familles ont été faits aussi sur quatre des variables étudiées pour leur anisotropie; ils devront être généralisés aux autres variables. Pour le moment, seules les caractéristiques suivantes ont été examinées : le rendement en cellulose, la longueur moyenne des fibres, le pourcentage de résine et la densité globale. Seule, cette dernière grandeur peut être considérée comme étant différemment anisotrope suivant les familles, ce résultat n'étant significatif qu'à $5 \%$ pour les mesures effectuées sur les carottes droites et en-dessous de la limite de la signification, au même seuil, dans le cas des carottes obliques prélevées sur des demi-frères des mêmes familles.

L'anisotropie est-elle héritable? Un tel phénomène a déjà été signalé à propos de l'anisotropie des largeurs de cernes (Polge et Illy, 1967, op. cit. - Polge et Illy, 1968).

Il est possible que, comme dans le cas des largeurs de cernes, d'autres anisotropies conduisent à des héritabilités significatives, en particulier celles qui sont relatives à la densité et à ses composantes. Des calculs étudiant la validité de cette hypothèse sont en cours.

\section{3. - TRANSMISSION HÉRÉdTTAIRE DES CARACTÉRES, ÉTUDIÉE A PARTIR DES RÉSULTATS DU TEST D 441 ET DES ARBRES-MÊRES}

L'estimation de la façon dont on trouve chez les descendants des analogies, soit entre eux à l'intérieur des familles auxquelles ils appartiennent, soit avec les propriétés des arbres adultes dont ils sont issus, peut se faire au moyen de deux types de calculs. L'un d'entre eux permet de mettre au jour des caractères de famille uniquement à partir de valeurs observées sur les descendants de diverses origines grâce à l'analyse de variance. L'autre compare plus directement et d'une façon plus parlante à l'esprit, les valeurs des caractères mesurés sur les arbres-mères aux valeurs trouvées chez leurs descendances; la difficulté vient ici du fait que, chez ces dernières, les constituants du bois sont à un stade juvénile évoluant d'année en année et que par conséquent les valeurs des caractères mesurés ne sont que provisoires puisqu'elles tendent vers un état stable mais lointain, celui des adultes.

\section{1. - Héritabilité calculée par analyse de variance sur les caractères juvéniles des demi-frères du test D 441}

Ce mode d'étude de la transmission des caractères ne fait aucune référence aux résultats relatifs aux arbres-mères; on y cherche en quelque sorte à mettre en évidence le fait que les 
individus appartenant à une même famille se ressemblent plus entre eux que ne font des descendants issus de familles différentes. A cet effet, une analyse de variance sur les arbres du bloc incomplet équilibré à 41 familles a déterminé d'une part, la variance entre les familles, $\sigma_{\mathrm{F}}^{2}$, qui a dans cette étude 40 degrés de liberté, et d'autre part, la variance résiduelle des individus dans les familles, $\sigma_{n}^{*}$, qui a 369 degrés de liberté. Cette dernière quantité est la résultante de plusieurs facteurs dont, en particulier, l'influence du milieu. La combinaison de ces deux données permet d'estimer le coefficient de corrélation intraclasse par l'expression

$$
p=\frac{\sigma_{F}^{2}}{\sigma_{f}^{2}+\sigma_{k}^{2}}
$$

qui représente la part de la variance entre familles par rapport à la variance totale évaluće dans le dispositif en question. Comme le test D 441 est supposé être constitué de demi-frères vrais (G. ILly, 1966, op. cit.), l'héritabilité des divers caractères vaut $h^{2}=4 \rho$ (ARBEZ et MiLLier, 1970).

L'existence de cette héritabilité est considérée comme acquise lorsque le test F correspondant est significatif; elle ne devrait normalement pas dépasser la valeur 1 , mais il arrive que des caractères mesurés de façon peu précise conduisent à des valeurs sans signification supérieures à 1 ou même inférieures à 0 . Les degrés de liberté du test F sont 40 et 369 ; malheureusement ils ne conviennent que dans le cas oủ on dispose de valeurs pour 10 individus de

TABLEAU 6 - TABLE 6

Hérifabilité calculée à partir de lanalyse de variance $\mathrm{h}^{2}=4 \frac{\sigma^{2} \mathrm{~V}}{\sigma^{2}{ }_{\mathrm{P}}+\sigma^{2}}$

Heritability calculated by the way of an analyse of variance.

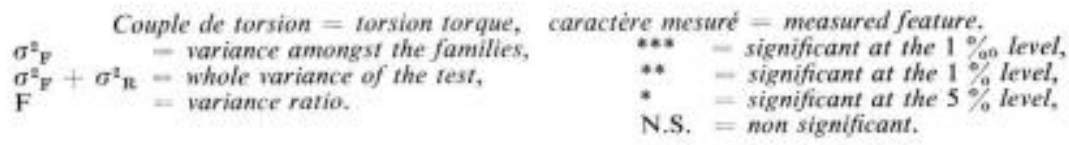

\begin{tabular}{|c|c|c|c|c|c|c|c|c|}
\hline \multirow[t]{2}{*}{ Caractère mesuré } & \multicolumn{2}{|c|}{$\begin{array}{c}\text { Carottes droites } \\
\text { E }\end{array}$} & \multicolumn{2}{|c|}{$\begin{array}{c}\text { Carottes droites } \\
\text { W }\end{array}$} & \multicolumn{2}{|c|}{$\begin{array}{c}\text { Carottes obliques } \\
\text { E }\end{array}$} & \multicolumn{2}{|c|}{$\begin{array}{c}\text { Carottes obliques } \\
\text { W }\end{array}$} \\
\hline & $h^{2}-4 e$ & $F_{369}^{40}$ & $h^{2}=40$ & $F_{369}^{40}$ & $h^{2}=4 \varrho$ & $F_{369}^{40}$ & $h^{2}-4 e$ & $\mathrm{~F}_{369}^{40}$ \\
\hline Infradensité $\ldots . . . \ldots \ldots$ & 0,432 & $2,22 * * *$ & 0,520 & $2,51 * *$ & 0,264 & $1,70^{* *}$ & 0,524 & $2,51 * * *$ \\
\hline Teneur en résine..... & $(1,440)$ & $6,63 * * *$ & $\longrightarrow$ & & 0,568 & $2,66 * * *$ & 0,012 & N,S \\
\hline $\begin{array}{c}\text { Longueur moyenne des } \\
\text { fibres } \ldots \ldots \ldots \ldots \ldots\end{array}$ & & & & & $(-0,096)$ & N.S. & 0,144 & $\begin{array}{l}1,38^{*} \text { a } \\
\text { la limite }\end{array}$ \\
\hline Rendement en fibres ... & & & 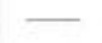 & $\longrightarrow$ & 0,056 & N.S. & $(-0,032)$ & N.S. \\
\hline Angle de la fibre torse... & 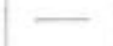 & - & 0,012 & N.S. & - & 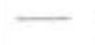 & $(-0,072)$ & N.S. \\
\hline $\begin{array}{l}\text { Couple de torsion au } \\
\text { moment du sondage.. }\end{array}$ & & & 0,156 & $1,41^{*}$ & - & - & 0,068 & N.S. \\
\hline
\end{tabular}


TABLEAU 7 - TABLE 7

Héritabilité calculée à partir de l'analyse de variance $\mathrm{h}^{2}=4 \frac{7}{\sigma^{2} y+\sigma_{\mathrm{R}}^{2}}$

Heritability calculated by the way of an analysis of nariance.

\begin{tabular}{|c|c|c|c|c|}
\hline \multirow[t]{2}{*}{ Caractère mesuré } & \multicolumn{2}{|c|}{ Carottes droites $\mathrm{E}$} & \multicolumn{2}{|c|}{ Carottes droites W } \\
\hline & $h^{2}-4 e$ & $F_{246}^{40}$ & $h^{2}=4 a$ & $F_{246}^{40}$ \\
\hline Infradensité $\ldots \ldots \ldots \ldots \ldots \ldots \ldots \ldots$ & 0,304 & $1,57^{*}$ & 0,500 & $2,01 * \cdots$ \\
\hline Teneur en résine $\ldots \ldots \ldots \ldots \ldots \ldots \ldots \ldots$ & $(1,464)$ & $5,04 * \cdots$ & - & - \\
\hline 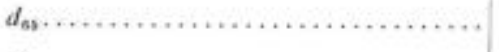 & 0,509 & $2,02 * *$ & 0,476 & $1,95 * * *$ \\
\hline 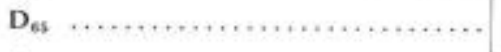 & 0,416 & $1,81 \cdots$ & 0,500 & $2,01 * * *$ \\
\hline$d_{6 \sigma}, \ldots \ldots \ldots \ldots \ldots \ldots \ldots \ldots \ldots \ldots \ldots$ & 0,716 & $2,53 * *$ & 0,780 & $2,71 \cdots$ \\
\hline 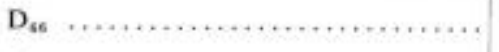 & 0,348 & $1,66^{*}$ & 0,272 & $1,52^{*}$ \\
\hline$I_{\mathrm{s}}, \ldots \ldots \ldots \ldots \ldots \ldots \ldots \ldots \ldots \ldots \ldots$ & 0,340 & $1,65^{*}$ & 0,004 & N.S. \\
\hline$I_{s 6} \ldots \ldots \ldots \ldots \ldots \ldots \ldots \ldots \ldots \ldots \ldots$ & 0,044 & N.S. & 0,476 & $1,95 * * *$ \\
\hline$\% l d \geqslant 350_{6} \quad \ldots \ldots \ldots \ldots \ldots \ldots \ldots \ldots$ & 0,332 & $1,63^{*}$ & 0,448 & $1,89 * * *$ \\
\hline$\% t d \geqslant 350_{s e} \ldots \ldots \ldots \ldots \ldots \ldots \ldots \ldots$ & 0,512 & $2,03 * *$ & 0,280 & $1,53^{*}$ \\
\hline$\% l d \geqslant 400_{6 s} \quad \ldots \ldots \ldots \ldots \ldots \ldots \ldots \ldots$ & 0,312 & $1,59 *$ & 0,364 & $1,70^{* \bullet}$ \\
\hline$\% l d \geqslant 400 \omega \ldots .$. & 0,644 & $2,34^{* *}$ & 0,236 & $1,44^{*}$ \\
\hline$\% l d \geqslant 450_{4 s} \ldots \ldots \ldots \ldots \ldots \ldots \ldots \ldots$ & 0,460 & $1,91^{* *}$ & 0,312 & $1,60^{* *}$ \\
\hline$\% l d \geqslant 450_{s e}, \ldots \ldots \ldots \ldots \ldots \ldots \ldots$. & 0,288 & $1,55^{*}$ & 0,380 & $1,74^{* *}$ \\
\hline $\mathbf{D}_{\mathrm{ss}}-d_{\mathrm{s}} \ldots \ldots \ldots \ldots \ldots \ldots \ldots \ldots$ & 0,768 & $2,67 * * *$ & 0,764 & $2,66^{* * *}$ \\
\hline $\mathbf{D}_{\mathrm{s}}-d_{\mathrm{s}} \ldots \ldots \ldots \ldots \ldots \ldots \ldots \ldots$ & 0,476 & $1,95 * * *$ & 0,468 & $1,92 * \bullet \bullet$ \\
\hline
\end{tabular}

chacune des 41 familles et que par conséquent l'analyse de variance a été faite suivant le schéma correspondant à celui des blocs incomplets équilibrés. Dans cette expérience de tels résultats ne sont disponibles que dans le cas des caractéristiques suivantes : infradensité, teneur en résine, couple de torsion au moment des sondages, angle de la fibre torse mesurée à partir des carottes de sondage, longueur moyenne des fibres et rendement en fibres, pour les carottes droites et (ou) obliques et pour les orientements est et (ou) ouest. Dans le cas de tout ce qui concerne les composantes de la densité, seules les 7 meilleures images radiographiques des sondages droits ont été retenues parmi les 10 théoriquement disponibles; il en résulte qu'une analyse en blocs incomplets équilibrés devient impossible et que le nombre de degrés de liberté passe de 369 à 246 ce qui nécessite des valeurs $\mathrm{F}$ plus élevées pour atteindre un seuil de signification statistique donné. 
Le tableau 6 résume les principaux résultats obtenus. L'infradensité jouit d'une très bonne héritabilité, les $\mathrm{F}$ correspondant aux différents cas étant significatifs à $1 \%$ ou même $1 \%$ o-

La teneur en résine fournit en revanche des résultats fort instables du fait des difficultés expérimentales déjầ mentionnées et même sans signification (même si le test $\mathrm{F}$ semble indiquer le contraire); il semble que la valeur $h^{2}=0,568$ avec un test $\mathrm{F}$ de 2,66 ait, elle, une signification normale. Les côtés ouest ne donnent aucun résultat positif en ce qui concerne cette grandeur. Les deux seuls autres résultats significatifs ont été trouvés dans le cas du côté ouest : il s'agit d'une part du couple de torsion systématiquement mesuré au moment du sondage sur ce côté des arbres (H. Polge, R. Kelter, 1970) et pour lequel on trouve $h^{2}=0,156$ ce qui correspond à un $\mathrm{F}$ de 1,41 significatif au seuil de $5 \%$ et de la longueur moyenne des fibres pour qui F vaut 1,38 (donc un peu inférieur au seuil de $5 \%$ ) et $h^{2}=0,144$.

Les héritabilités de l'infradensité et de la teneur en résine ont été à nouveau calculées dans le cas des sept échantillons pris parmi les dix, en plus des héritabilités des composantes de la densité qui, comme le montre le tableau 7, sont élevées et stables quel que soit l'orientement.

\section{2. - Héritabilité calculée à partir des coefficients de régression entré les caractères des arbres-mères et ceux des demi-frères du test D 441}

L'utilisation du coefficient de régression est un des moyens d'apprécier les liaisons qui peuvent exister entre des caractéristiques homologues relevées chez les arbres adultes et leurs descendants. Si l'on appelle $X_{i}$ les valeurs du caractère $i$ mesuré sur les arbres-mères et $Y_{i}$ celles du caractère analogue de leurs descendants demi-frères $\left(Y_{i}\right.$ est une valeur moyenne obtenue à partir de 7 ou 10 échantillons par famille suivant les cas) l'héritabilité $h_{1}^{\prime}$ d'une génération à l'autre est prise égale au double du coefficient de régression des $\mathrm{Y}_{i}$ en $\mathrm{X}_{i}$ soit

$$
h_{1}^{2}=2 b \text { où } b=r \frac{\sigma_{Y_{4}}}{\sigma_{X_{i}}} \text { et } r=\frac{\text { covariance }\left(\mathrm{X}_{i}, \mathrm{Y}_{i}\right)}{\sqrt{\text { variance } \mathrm{X}_{i} \times \text { variance } \mathrm{Y}_{i}}}
$$

Le test de signification consiste à comparer, en en faisant le rapport, $h_{1}^{2}$ à son écart type. La variance de $b$ vaut

$$
\sigma_{1}^{2}=\frac{1}{n-2} \cdot \frac{\sigma_{\mathrm{v}_{\mathrm{t}}}^{2}}{\sigma_{\mathrm{x}_{1}}^{2}}
$$

I'écart-type de $h_{4}^{2}$ vaut deux fois celui de $b$, soit

$$
\sigma_{h_{4}^{2}}=2 \sigma_{\mathrm{b}}=2 \sqrt{\frac{1}{n-2} \cdot \frac{\sigma_{Y_{i}}}{\sigma_{\mathrm{x}_{4}}}}
$$

(W. A. BECKER, 1968). Il suffit done de calculer la quantité $h_{i}^{t}$ et de voir quand elle dépasse les valeurs 2,04 à 2,06 suivant le nombre de couples (au seuil de signification de $5 \%$ ) ou 2,76 à 2,78 (seuil de signification de $1 \%$ ); les nombres de couples disponibles sont de 28 à 33 , certaines caractéristiques étant absentes dans l'une des deux populations.

Les calculs de régression ont été faits sur 13 variables classiques ainsi que sur 8 composantes de la densité. D'une façon évidente (tableau 8) les résultats provenant de ce type de 
TABLEAU 8 - TABLE 8

Héritabilité calculée à partir des régressions entre les descendants du test $\mathrm{D} 441$ et les arbres-mères : $\mathrm{h}^{2}=2 \mathrm{~b}$

Heritability calculated by the way of the regressions befween the progenies of the test D 441 and their mother-trees.

variables comparées = compared variables, écorce et caur mélangés = bak side plus pith side,

orientement $=$ orientation, cart moyen entre... - mean difference between.

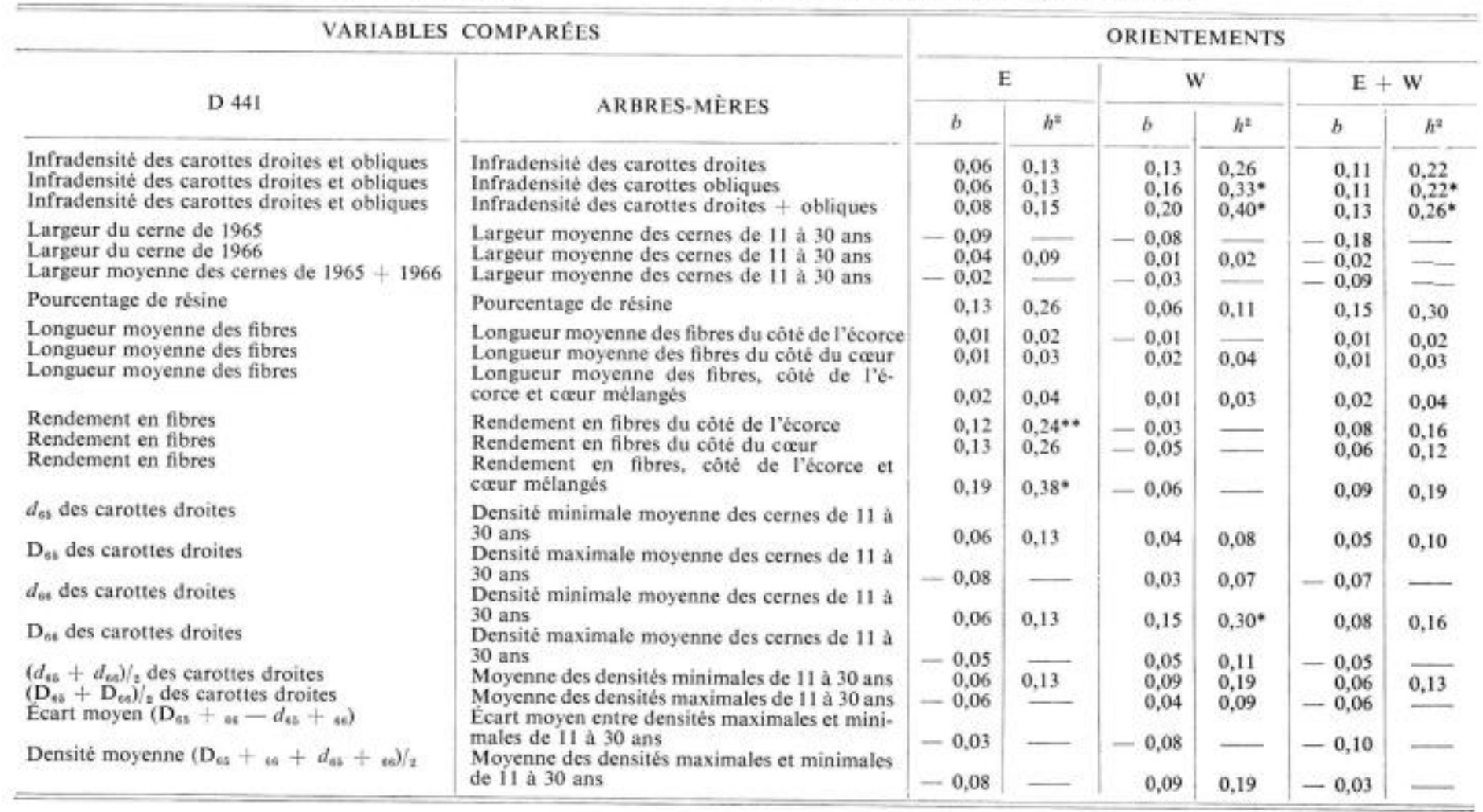


traitement ne sont significatifs qu'avec une fréquence bien moindre que dans le cas des calculs portant sur les seuls descendants. En effet, l'héritabilité calculée par analyse de variance est tirée d'une collection de familles regroupées au même endroit, donc soumises aux mêmes conditions de milieu, celles du dispositif statistique utilisé, qui diffèrent de chacunes de celles qui sont propres à chaque arbre-mère; ceci s'ajoute aux disparités entre les valeurs moyennes des caractéristiques qui sont dues aux différences d'âge entre les deux populations, à l'instabilité des propriétés du bois juvénile et il en résulte des liaisons lâches et des héritabilités plus rarement significatives. Parfois les calculs ont abouti à des valeurs négatives pour $b$, ce qui se produit lorsque la covariance est négative (le coefficient de corrélation entre les $X_{i}$ et les $Y_{i}$ est lui-même négatif); l'héritabilité ne peut, dans ces conditions, être définie et le tableau ne porte que la valeur de $b$.

Parmi les huit composantes de la densité envisagées, seule une a donné un résultat significatif au seuil de $5 \%$, avec une héritabilité de 0,30 dans le cas de l'orientement ouest constitué de bois considéré comme normal : il s'agit de la densité minimale de l'année 1966 des descendants vis à vis de la densité minimale moyenne des 20 cernes de 11 à 30 ans des arbres-mères. Aucune des autres composantes ou combinaison de composantes, comme l'écart entre les densités maximales et minimales, $\mathrm{n}$ 'a donné une héritabilité significative; très souvent même les coefficients $b$ sont négatifs.

Chez les variables classiques on n'a trouvé que deux groupes à avoir fourni des résultats positifs, ce sont d'une part les infradensités relatives aux côtés ouest et aux moyennes entre les côtés est et ouest et d'autre part les rendements en fibres des còtés est. L'héritabilité de l'infradensité dans l'orientement ouest est comprise entre 0,26 et 0,40 , les résultats étant significatifs au seuil de $5 \%$; pour la moyenne des deux orientements, $h^{2}$ varie de 0,22 à 0,26 avec la même précision. Ce sont les rendements en fibres qui donnent les deux dernières héritabilités significatives avec des valeurs de 0,24 (au seuil de $1 \%$ ) dans le cas des échantillons du test D 441 et du bois formé au voisinage de l'écorce chez les arbres-mères, ou de 0,38 (seuil de $5 \%$ ) si l'on prend chez ces derniers la moyenne entre les rendements du bois proche de l'écorce et du bois proche du cour.

D'autres calculs d'héritabilité par régression sont en projet sur les composantes de la densité et en particulier sur les pourcentages de bois de densité supérieure à des valeurs données et les pentes des courbes microdensitométriques entre divers niveaux de densité. La difficulté dans ces sortes d'études provient du fait qu'il y a beaucoup de différence entre la structure des courbes densitométriques des arbres adultes et de celles des jeunes arbres. II faut tenter de définir des niveaux qui pourraient se correspondre dans les deux populations (on peut penser par exemple au pourcentage de bois de densité supérieure à la moyenne entre les densités maximales et minimales, bien que la valeur absolue de cette moyenne ne soit pas identique chez des arbres âgés et des arbres jeunes), mais ceci présente les inconvénients déjà évoqués du fait des différences de moyennes et variances entre les deux termes de comparaisons.

Pour tenter de remédier à cette dernière difficulté ( $\propto$ effet d'échelle $»)$, le chapitre 43 présentera ci-dessous des héritabilités calculées à partir des coefficients de corrélation $r$ observés entre des caractères des arbres-mères et des caractères des descendants (FREY K. J. et Horner T., 1957, Steinhoff R. J. et Hoff R. J., 1971).

En effet, « pour tenir compte des différences de variances entre les arbres-mères (mesurés à l'åge adulte en forêt) et leurs descendants (mesurés au stade juvénile), on est amené 
(ARBEZ M., 1972) « à pondérer la valeur du coefficient de régression $b_{\mathrm{yx}}$ par le rapport $\frac{\sigma_{x_{1}}}{\sigma_{y_{1}}}$ de l'écart-type des parents à celui de leurs descendants ", et on définit l'héritabilité par :

$$
h^{2}=2 b_{\mathrm{vx}} \frac{\sigma_{\mathrm{x}_{r}}}{\sigma_{\mathrm{r}_{\mathrm{r}}}}
$$

dont la valeur numérique n'est autre (dans ce cas particulier) que celle du double du coefficient de corrélation $r$ entre parents et descendants.

\section{4. - CALCULS DE CORRÉlATION RELATIFS AUX CARACTÉRISTIQUES DES ARBRES DU TEST D 441 ET DES ARBRES-MÊRES}

\section{1. - Corrélations entre les variables mesurées chez les descendants du test D 441}

Le tableau 9 à 33 lignes et 33 colonnes donné en annexe porte les coefficients calculés dans le cas des 31 familles de descendants correspondant aux arbres-mères pour lesquels on dispose de données complètes. Il contient 24 lignes et colonnes de coefficients trouvés à partir des caractéristiques mesurées sur les carottes de sondage ainsi que 9 lignes et colonnes supplémentaires où sont placées les variables qui décrivent la croissance des jeunes arbres au cours des années 1967 et 1968 .

Les densités minimales $d$ sont fortement liées au pourcentage $l d \geqslant 350 \mathrm{~g} / \mathrm{dm}^{3}$ de façon positive, mais il semble qu'il existe, pour 1966, un antagonisme entre densité minimale et diamètre à mi-hauteur en 1968. Ainsi que l'on peut s'y attendre, les densités maximales D sont plus liées avec le pourcentage $l d \geqslant 450 \mathrm{~g} / \mathrm{dm}^{3}$ qu'avec ceux des niveaux inférieurs. $d_{65}$ et $d_{66}$ sont en corrélation, de même que $\mathrm{D}_{65}$ et $\mathrm{D}_{66}, I_{65}$ et $l_{66}$. Ces deux dernières variables sont aussi en relation avec les valeurs des volumes des deux saisons de végétation de 1967 et 1968.

Les hauteurs totales leur sont liées positivement, ce qui traduit probablement un effet de la vigueur de croissance.

L'hétérogénéité du bois varie dans le mème sens que le pourcentage $l d \geqslant 450 \mathrm{~g} / \mathrm{dm}^{3}$, parce que, sans doute, elle dépend plus des densités élevées, en particulier des maxima; elle n'est que peu ou pas liée aux densités minimales. A l'intérieur d'une même saison de végétation, les liens entre les diverses largeurs de bois des niveaux successifs de densité repérés sont élevés, et plus que ceux qui existent entre mêmes niveaux, mais entre deux saisons qui se suivent, ainsi qu'on peut le voir en comparant les chiffres situés aux intersections des lignes et des colonnes 7 à 12. La liaison la plus élevée se trouve en 1965 entre $I_{65} 350 \mathrm{~g} / \mathrm{dm}^{3}$ et $l_{65}$ $400 \mathrm{~g} / \mathrm{dm}^{3}$, pour le côté ouest, avec un coefficient $r=0,889$. Les liaisons entre les pourcentages correspondants sont également étroites; cela suggère une fois de plus l'intérêt qu'il y aura à encore mieux définir la forme des courbes densitométriques et d'étudier leurs pentes entre les divers niveaux.

L'infradensité semble être plus souvent en liaison avec les niveaux de faible densité qu'avec ceux de forte densité; elle est en corrélation négative avec la longueur des fibres, ellemême liée négativement avec diverses composantes de la densité comme on peut le voir ligne 15 et colonne 13. Le rendement en fibres n'est lié à aucun facteur si ce n'est avec le pourcentage de résine du côté est $(r=-0,439)$. 
Les caractéristiques de croissance sont surtout liées aux largeurs et de façon positive. Le diamètre à mi-hauteur et le volume de l'année 1968 sont en antagonisme avec les densités minimales (colonnes 1, 3, 16). La note de rectitude pour 1967 (qui va de 1 à 5 et est d'autant plus faible que l'arbre est moins droit) n'est en corrélation qu'avec la même variable pour 1968 , alors que $l_{66}, l_{66} 350 \mathrm{~g} / \mathrm{dm}^{3}$ et $l_{66} 400 \mathrm{~g} / \mathrm{dm}^{3}$ sont en opposition significative avec le fait que les tiges sont uniques (notées : 1 ) ou fourchues (notées : 2) ainsi qu'il ressort des colonnes 6,8 , et 10 et de la ligne 30 dans le cas de l'orientement ouest. La note de rectitude de 1968 est en corrélation positive, mais assez faible, avec la hauteur totale des arbres mesurée la même année. Hauteurs et diamètres varient dans le même sens pour une même saison de végétation et sont liés d'une année à l'autre. On retrouve un phénomène qui fait penser à un résultat déjà obtenu (H. Polge, G. ILly, 1968 op. cit.) : dans le cas de 7 familles, ces derniers ont trouvé une liaison positive élevée entre la largeur du cerne de l'année 1965 et la densité globale de ce même cerne. Cette corrélation génotypique est particulièrement intéressante puisqu'elle montre qu'il n'y a pas, chez le pin maritime, d'opposition entre une croissance rapide et une forte densité de la matière ligneuse. Dans le cas des 31 familles étudiées ici, les largeurs des cernes de 1965 et 1966 ne sont pas liées directement à l'infradensité mesurée sur toute la carotte, done de la moelle à l'année 1966, mais $\left(I_{65}+I_{66}\right) / 2$ est en corrélation positive avec $d_{\mathrm{Bg}}\left(r=0,38\right.$ pour le côté est), $l_{\mathrm{GG}} 400 \mathrm{~g} / \mathrm{dm}^{3}(r=0,43$ pour la moyenne des côtés est et ouest) et $l_{\mathrm{Bg}} 450 \mathrm{~g} / \mathrm{dm}^{3}(r-0,49$ pour le côté est). Ce fait indique que les arbres à forte croissance sont ceux qui forment aussi des cernes où la zone de bois à densité élevée $\left(400 \mathrm{~g} / \mathrm{dm}^{3}\right)$ est importante.

\section{2. - Corrélations entre les variables mesurées chez les arbres-mères}

Les densités minimales moyennes de 11 à 30 ans sont liées positivement (tableau 10 en annexe) aux divers pourcentages $l d \geqslant$ à une valeur donnée, en particulier à $\% / d \geqslant 400 \mathrm{~g} / \mathrm{dm}^{3}$ $\left(r=0,914\right.$ pour la moyenne des côtés est et ouest) et à \% $\%$ ld $\geqslant 900 \mathrm{~g} / \mathrm{dm}^{3}(r=0,743$ dans les mêmes conditions); elles sont aussi en corrélation positive avec les diverses infradensités calculées. En revanche $d$ et $\mathrm{D}-d$ n'ont aucun lien apparent. A l'inverse de ce qui se passe chez les descendants du test D 441, lorsque des liaisons existent entre ces deux variables, $d$ et $l$ varient ici en sens contraire.

Ce qui est vrai pour $d$ l'est aussi pour $\mathrm{D}$, son coefficient de corrélation avec $\% l d \geqslant 800 \mathrm{~g} / \mathrm{dm}^{3}$ atteint 0,913 , à l'exception de ce qui a trait à $\mathrm{D}-d$.

L'hétérogénéité est liée positivement à $\mathrm{D}, r=0,741$, comme dans le cas des jeunes descendants, alors qu'on n'a trouvé aucun lien avec $d$.

La largeur moyenne des cernes, $/$ de 11 à 30 ans, est en corrélation négative avec toutes les autres caractéristiques, composantes de la densité, hétérogénéité (côté est) et même, longueurs de fibres et rendement en fibres (côté est).

Les infradensités mesurées et $\% l d \geqslant 400 \mathrm{~g} / \mathrm{dm}^{3}, \% l d \geqslant 500 \mathrm{~g} / \mathrm{dm}^{3}, \% l d \geqslant 600 \mathrm{~g} / \mathrm{dm}^{3}$ sont en liaison étroite (le coefficient $r$ peut atteindre 0,894). L'hétérogénéité $\mathrm{D}-d$ du côté est peut être liée au rendement en fibres, celui-ci variant comme la longueur des fibres (colonne 18 et ligne 19, colonne 19 et ligne 20, colonne 22 et ligne 23). Un fait qui semble important à retenir de ce tableau est le suivant : si l'hétérogénéité du bois est fortement en 
corrélation avec les densités maximales, comme on I'a vu, elle semble en revanche n'avoir aucun rapport avec l'infradensité. II n'y aurait donc pas incompatibilité entre haute densité globale et homogénéité.

\section{3. - Corrélations entre les caractéristiques des arbres-mères et celles de leurs descendants du test D 441}

Le tableau 11 à 23 lignes (arbres-mères) et 27 colonnes (D 441) donné en annexe présente des coefficients de corrélation entre tous les caractères de l'une et l'autre des populations. Une première remarque peut être faite : les coefficients relatifs aux variables de croissance des descendants et aux variables des arbres-mères sont tous négatifs à l'exception de ce qui a trait à la hauteur et au volume de 1967 (colonnes 19, 22, ligne 16).

En particulier, les volumes des jeunes arbres sont en corrélation négative avec la densité maximale moyenne D 11 à 30 ans ainsi qu'avec le pourcentage $l d \geqslant 800 \mathrm{~g} / \mathrm{dm}^{3}$, la quantité $(\mathrm{D}+d) /_{2} \mathrm{et}$, à un degré moindre, avec l'hétérogénéité $\mathrm{D}-d$ des 20 années considérées.

De très nombreuses composantes de la densité des descendants ont des liaisons négatives avec des caractères des arbres-mères tels que les longueurs de fibres, les rendements en fibres ou, ce qui est plus surprenant, avec leurs composantes de la densité comme le montre le comportement relatif de $\mathrm{D}_{65}$ vis-à-vis des pourcentages $l d \geqslant 900 \mathrm{~g} / \mathrm{dm}^{3} \mathrm{et} l d \geqslant 1000 \mathrm{~g} / \mathrm{dm}^{3}$.

Il faut souligner en revanche que le rendement en fibres des descendants est en liaison positive (pour le côté est) avec certaines caractéristiques de leurs parents : la densité moyenne $d 11$ à 30 ans, la longueur moyenne des fibres du côté de l'écorce (côté est et moyenne est plus ouest), le rendement en fibres pour la zone du cœur ou pour l'ensemble des côtés du cơur et de l'écorce.

Le tableau laisse apparaitre un vide à l'intersection des lignes et colonnes des pourcentages de bois de densité supérieure à certains niveaux. Les différences d'âge et de milieu entre les deux populations ont dû en ce cas jouer un rôle prépondérant. II existe quelques liens positifs entre caractéristiques non homologues de densité. Par exemple, on trouve que $d_{65} \mathrm{et}$ $d_{\text {EG }}$ sont en corrélation positive avec le pourcentage $l d \geqslant 300 \mathrm{~g} / \mathrm{dm}^{3}$ (et pas avec $d 11$ à 30 ans comme on l'a vu); $d_{66}$ est lié à l'infradensité de 11 à 30 ans (côté ouest), à l'infradensité globale des carottes obliques (côté est plus côté ouest) et à $(\mathrm{D}+d) / 211$ à 30 ans. Notons également que $\left(d_{65}+d_{66}\right) / 2$ varie comme $l d \geqslant 300 \mathrm{~g} / \mathrm{dm}^{3}$ ou l'infradensité des carottes obliques.

L'infradensité moyenne des carottes droites et obliques du test D 441 présente quelques relations positives avec des grandeurs homologues ou des composantes de la densité chez les arbres-mères : $d 11$ à 30 ans, D 11 à 30 ans, les pourcentages $l d \geqslant 400 \mathrm{~g} / \mathrm{dm}^{3}, l d \geqslant 500 \mathrm{~g} / \mathrm{dm}^{3}$ $\left(r=0,462\right.$ pour la moyenne est plus ouest), $l d \geqslant 900 \mathrm{~g} / \mathrm{dm}^{3},(D+d) / 211$ à 30 ans, l'infradensité de 11 à 30 ans ( $r=0,356$ pour la moyenne est plus ouest) ainsi que, comme on peut le voir sur la figure 4 , pour le côté ouest, avec l'infradensité globale moyenne des carottes droites et obliques $(r=0,366)$.

En doublant ces deux derniers coefficients, on trouve des quantités analogues aux héritabilités du type $h^{2}=2 r$ calculées par H. Polge et G. Illy $(1968, o p$. cit ) dans le cas de 7 familles de la même expérience (voir le tableau 12). Ces valeurs d'héritabilité de l'infradensité sont en effet $h^{2}=0,71$ pour l'infradensité moyenne des descendants et l'infradensité de 11 à 30 ans des arbres-mères, ou $h^{2}=0,73$ pour I'infradensité moyenne des descendants et 


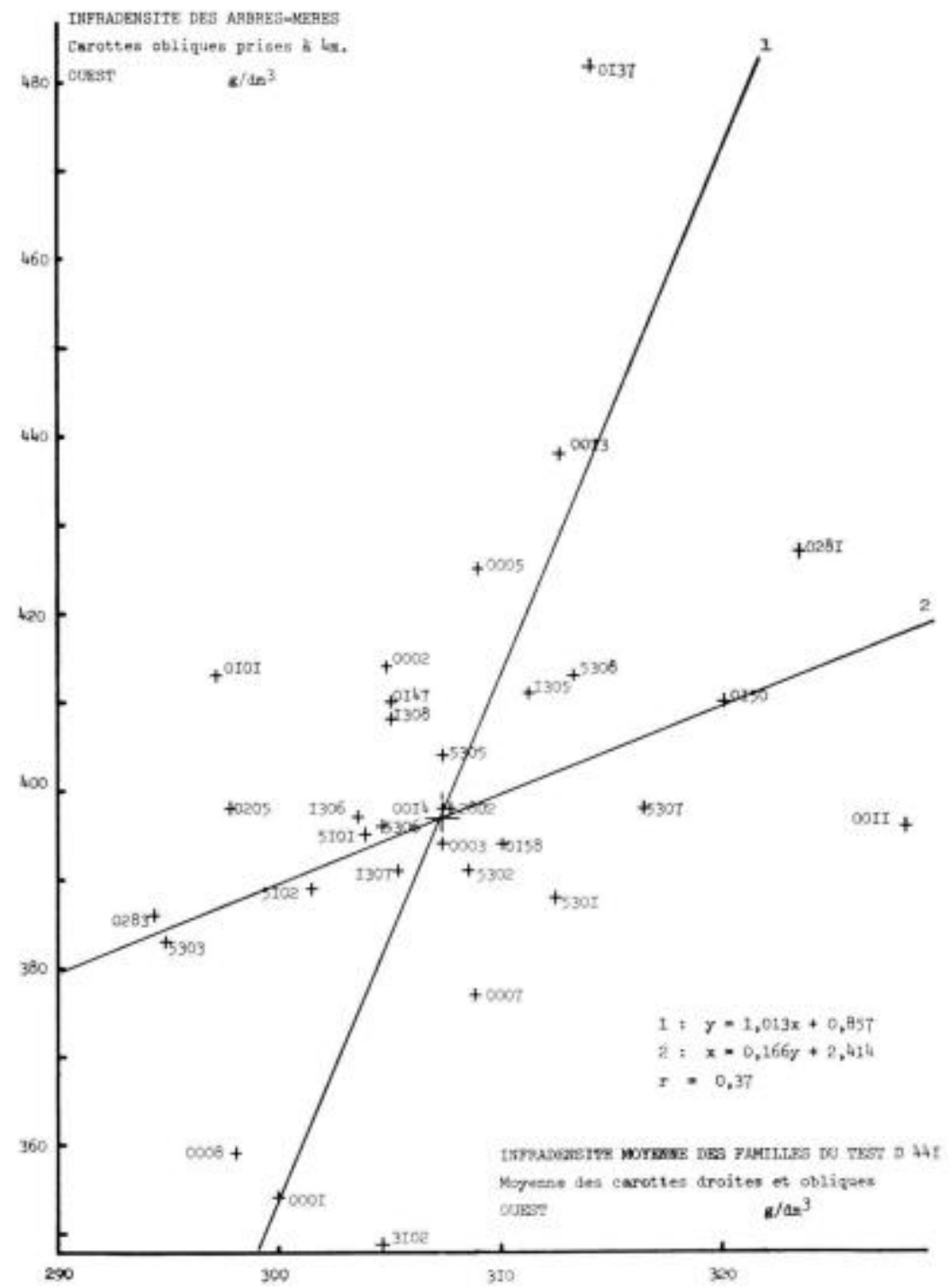

FiG. 4. - Corrélation entre les arbres-mères et la valeur moyenne de leurs descendants FIG. 4. - Correlation between the mother-trees and the mean values of their progenies

Infradensité des arbres-méres : mean specific graviry of the mother-trees.

Infradensite moyenne des familles du test D 441 ; mean specific gravity of the D 441 progeny test.

Carottes abliques prises a $4 \mathrm{~m}$ : slanting increment borings taken at the $4 \mathrm{~m}$ level.

Moyenne des carottes droites et obliques : average of the normal and slanting cores.

Ouest $=$ west.

l'infradensité globale moyenne des carottes droites et obliques des parents, qui sont très comparables à $h^{2}=0,72$ trouvée alors dans le cas des densités moyennes totales de 7 familles et 7 arbres-mères. 
Héritabillté $\mathrm{h}^{2}=2 \mathrm{r}$ calculée entre des caractères moyens des descendants et des caractères strictement homologues ou non de leurs arbres-mères. Heritability $\mathrm{h}^{2}=2 \mathrm{r}$ calculated between mean features of the progenies and exactly or not corresponding features of their mother-trees.

\begin{tabular}{|c|c|c|c|c|c|c|c|c|c|}
\hline & & 1 & 2 & 3 & 4 & 5 & 6 & 7 & 8 \\
\hline & $\begin{array}{l}\text { Arbres-mères } \\
\text { Descendants } \\
\text { du test D } 441\end{array}$ & $\begin{array}{l}\text { Infradensité } \\
\text { moyenne } \\
\text { totale }\end{array}$ & $\begin{array}{c}\text { Infradensité } \\
\text { moyenne } \\
\text { des cernes } \\
1946 \text { à } 1965\end{array}$ & $\begin{array}{c}\% l d \\
\geqslant 300 \mathrm{~g} / \mathrm{dm}^{2}\end{array}$ & $\begin{array}{c}\% l d \\
\geqslant 900 \mathrm{~g} / \mathrm{dm}^{3}\end{array}$ & $\begin{array}{c}\text { Infradensité } \\
\text { de } \\
11 \text { à } 30 \text { ans }\end{array}$ & $\begin{array}{l}\text { Infradensité } \\
\text { globale des } \\
\text { carottes } \\
\text { obliques }\end{array}$ & $\begin{array}{l}(\mathrm{D}+d) / 2 \\
\mathrm{de} \\
11 \text { a } 30 \text { ans }\end{array}$ & $\begin{array}{l}\text { Infradensité } \\
\text { globale } \\
\text { moyenne des } \\
\text { carottes droites } \\
\text { et obliques }\end{array}$ \\
\hline 1 & $\begin{array}{l}\text { Infradensité moyen- } \\
\text { ne totale } \ldots \ldots \ldots \ldots\end{array}$ & 0,72 & 0,74 & & & & & & \\
\hline 2 & $\begin{array}{l}\text { Infradensité moyen- } \\
\text { ne du cerne } 1965 \ldots\end{array}$ & 0,88 & & & & & & & \\
\hline 3 & $d_{e s} \ldots \ldots, \ldots, \ldots$ & & & 0,79 & & & & & \\
\hline 4 & $d_{s t} \ldots \ldots \ldots \ldots \ldots$ & & & 0,71 & & 0,86 & $0,90 \quad 0,83$ & 0,74 & \\
\hline 5 & $\left(d_{s}+d_{s u}\right) / 2 \ldots \ldots$. & & & $0,86 \quad 0,83$ & & & $0,70 \quad 0,80$ & & \\
\hline 6 & $\% l d \geqslant 350 \mathrm{~g} / \mathrm{dm}^{2}$. & & & & & & 0,72 & & \\
\hline 7 & $\begin{array}{l}\text { Infradensité moyen- } \\
\text { ne des carottes droi- } \\
\text { tes et obliques ...... }\end{array}$ & & & 0,75 & 0,71 & 0,90 & & 0,92 & 0,75 \\
\hline
\end{tabular}

Pour les lignes et les colonnes 1 et 2 , le calcul a porté sur 7 familles. - 1 . For the lines and columns 1 and 2 , the calculation has been made on 7 families. Pour les lignes 3 à 7 et les colonnes 3 à 8, le calcul a porté sur 31 familles (côté est) ou 30 familles (côté ouest et moyenne est plus ouest). Dans ce cas, la disposition des chiffres est la suivante :

$$
\begin{aligned}
& \begin{array}{l|l|l}
\hline \text { A } & \text { - en A héritabilité relative au côté est } \\
\hline \text { B } & \text { - en B héritabilité relative au côté ouest }
\end{array} \\
& \text { - en C héritabilité relative à la moyenne entre les côtés est et ouest. }
\end{aligned}
$$

2. For the lines and columns 3 to 7 , and the columns 3 to 8 , the calculation has been made on 31 families (east side) or 30 families (west side) and mean value between, east and west sides; in this case, the heritabilies are arranged as follow : in $A=$ heritability concerning the east side, in $B=$ heritability concerning the west side, in $C$ - heritability concerning the mean between east and west sides. 
S'il était permis de considérer comme homologues $d_{65}, d_{66},\left(d_{65}+d_{66}\right) / 2$ d'une part et le pourcentage $l d \geqslant 300 \mathrm{~g} / \mathrm{dm}^{3}$ et l'infradensité globale des carottes obliques (donc aux cernes plutôt extérieurs) d'autre part, on trouverait des héritabilités d'environ 0,80 et du mème ordre de grandeur que celle qui fut calculée sur 7 familles entre la densité moyenne du cerne de 1965 des descendants et la densité moyenne totale des parents, soit $h^{2}=0,72$.

\title{
CONCLUSION
}

Cette étude avait pour but de comparer une population d'arbres-mères à leurs descendants demi-frères. Le principal intérêt réside probablement dans les divers calculs d'héritabilité et de corrélations dont certains semblent être une confirmation indirecte de la liaison génétique positive évoquée plus haut : un lien existerait entre bois à forte densité et largeur du cerne, pour ce qui est des premières années de végétation (entre 11 et 30 ans on n'a plus trouvé quelque chose de comparable chez les arbres-mères); il est expliqué par l'aptitude du pin maritime à pousser pendant l'automne.

Les composantes de la densité ont permis de préciser ce comportement. Les héritabilités calculées sur elles lors des analyses de variance sur les descendants ont montré à nouveau que la densité globale est héritable et laisse entrevoir que l'hétérogénéité du bois l'est aussi et fortement. Ces caractéristiques commencent du reste à être prises en considération dans les programmes de sélection, ce qui ne fait que rendre plus nécessaires les études sur les facteurs de la qualité du bois, en particulier les composantes de la densité ou les pentes des courbes densitométriques.

Nous envisageons pour notre part de donner encore plus d'extension, lorsque cela sera possible, à ces calculs de l'héritabilité de telles variables, reflets de la qualité du bois, et de leur anisotropie.

Reçu pour publication en janvier 1973

\section{REMERCIEMENTS}

Cette étude a été entreprise avec l'aide de G. Iuır, alors responsable de l'unité Amélioration de la Station de Recherches Forestières de l'Hermitage près de Bordeaux. Nous lui devons de nombreux conseils et la faculté d'avoir pu prélever les échantillons des arbres-mères du massif landais et de leurs descendants dans les places d'expérience de cette unité, actuellement gérées par Ph. BARADAT.

Que les personnes de cette station qui nous ont facilité la tâche et aidé au cours des prélèvements sur le terrain soient remerciés.

Les calculs ont été faits par la Station de Biométrie du C.N.R.F. sous la direction de C. MiLLier.

\author{
SUMMARY
}

wood characteristics of Martime pine (Pimus Pinaster Ait.) THEIR VARIABILITY AND HERITABILITY

The wood quality of maritime pine (Pinus pinaster Ait.) may be appreciated through the measurement of many characteristics. On this study are considered the only ones which use non destructive testings, namely in the particular case $5 \mathrm{~mm}$ increment borings. 
Three kinds of sampling were utilized : mature mother-trees scattered in the whole Landes forest, seedlings of the open pollinated progeny test D 441 (half sibs of these mother-trees) grown in an incomplete balanced bloc experimental design.

On the mother-trees have been measured several densitometric characteristics : the mean specific gravity and the density components for rings (from the 11th to the 30 th year), a growth characteristic : the ringwidth, two pulp characteristics : the pulp yield and the fiber length, a morphological characteristic : the angle of spiral grain in relation with the tree axis. When possible, the anisotropy of these data has also been investigated.

The same measurements with the addition of resin yield have been made, with the necessary adaptations due to the size of the samples, on the D 441 half sibs.

In the mother-trees the wood heterogeneity quantified by the differences between the maximum and minimum annual values of density is an interesting data to differenciate the individuals. On the contrary the ring-widths are by no means discriminant. The anisotropy affects several data but is negligible for different levels of high density.

The results of the D 441 experiment deal with juvenile wood which change from year to year; its specific gravity is low and its heterogeneity increases because the maximum annual densities themselves increase more rapidly than the minimum densities. The variability within families and the variability with age is often high and does not allow to give definitive conclusions. The anisotropy starts from the first years of the life of the trees, with the exception of the data of the latewood for the two growing seasons which are studied here. Some difference appear between families for the anisotropy of the specific gravity.

Two different methods of estimation of heritability have been used : analysis of variance amongst the progenies and correlation between the mother-trees and the mean values of their progenies.

With the first way, the heritability is high for the density components, particularely the difference between maximum and minimum annual densities.

From the correlation studies it appears that the heritability is significant only for a minimum annual density, for the specific gravity and for the pulp yield.

At last have been investigated all the correlations between the available date of the D 441 progeny test and between the parent trees and their families.

\title{
ZUSAMMENFASSUNG
}

\author{
UNTERSUCHUNGEN ÜBER DIE HOLZEIGENSCHAFTEN DER SEESTRANDKIEFER \\ (Pinus pinaster Ait.) : \\ VERÄNDERLICHKETT UND ERBFÄHIGKEIT
}

Die Holzqualitat kann bei der Seestrandkiefer Pinus pinaster durch die Messung zahlreicher Merkmale gewertet werden. In der vorliegenden Arbeit wurden nur diejenigen in Rücksicht genommen, die durch nicht zerstörende Vorgänge messbar waren, in erster Linie Probeaufnahmen mit dem $5 \mathrm{~mm}$ Presslerchen Zuwachsbohrer.

Die Stichproben haben einen zweifachen Ursprung : reife Bäume (Mutterbäume) auf dem ganzen "Landes " -Gebiet zerstreut, Nachkömmlinge aus Samen der ersteren (Halbbrüder im Versuch D 441) in einer statistichen Anlage.

Auf den Mutterbäumen wurden Messungen unternommen und zwar microdensitometrische Merkmale, Raumdichte und Dichtekomponenten für die zwanzig Jahre zwischen dem 11. und dem 30. Jahr, als Merkmal des Zuwachses, Jahrringbreite, zwei Merkmale für die Erzeugung von Papier. Faserertrag und Faserlänge, ein morphologisches Merkmal, Drehwuchs. Wenn es möglichwar, hat man auch die Anisotropie dieser Merkmale je nach der westlichen und östlichen Himmelsrichtung untersucht.

Die selben Messungen in Verhältnis gebracht zu der Grösse der Proben wurden bei den Halbbrûdern aus dem Versuch D 441 unternommen. Dazu kommt der Ertrag an Harz.

Was die Mutterbäume betrifft, ergibt sich dass die durch den Unterschied zwischen den Werten der maximalen und minimalen Dichte gemessene Heterogeneität ein interessantes Merkmal ist, womit man die einzelnen Individuen unterscheiden kann. Die Jahrringbreite hingegen liefert kein Mittel zur 
Unterscheidung. Bei vielen Merkmalen erscheint die Anisotropie; sie ist jedoch abwesend bei gewissen starken Dichteniveaus.

Die Ergebnisse des Versuchs D $44 I$ gründen sich auf Merkmalen des jugendlichen Holzes, das sich von Jahr zu Jahr ändert, mit schwacher Raumdichte und dessen Heterogeneitait zunimmt, weil die Werte der maximalen Dichten schneller wachsen als diejenigne der minimalen Dichten. Die Veränderlichkeit der Ergebnisse kann sehr gross sein innerhalb der Familien oder auch mit der Zeit und verbieten es zu endgültigen Schlüssen zu gelangen. Die Anisotropie wird schon in den ersten Lebensjahren der jüngen Baùme festgestestellt mit Ausnahme der Spätholzzonen der zwei untersuchten Wachstumzeiten. Anisotropische Unterschiede bestehen zwischen den Familien in Hinsicht auf die Raumdichte.

Rechnungen über die Erbfähigkeit (Heritabilitāt) wurden nach zwei Methoden unternommen und zwar durch die Analyse der Veränderlichkeit der Variablen bei den Familien von Nachkömmlingen oder durch die Korrelation zwischen den Mutterbäumen und den Halberüdern. Im ersteren Falle erschienen die Dichtekomponenten und namentlich der Unterschied zwischen maximaler und minimaler Dichte als besonders erblich. Im zweiten Falle haben die Korrelationsrechnungen nur für eine minimale Dichte, für die Raumdichte und für einen Faserertrag Heritabilitäten erscheinen lassen.

Allgemeine Korrelationsrechnungen betreffen alle verfügbaren Variablen des Versuchs D 44I. der Mutterbäume, sowie die paarweise zusammengestellten Ergebnisse für Mutterbaūme und Nachkömmlinge,

\section{RÉFÉRENCES BIBLIOGRAPHIQUES}

Arafz M. - Notions de génétique des populations et de génétique quantitative. Station d'Amélioration des Arbres Forestiers. I.N.R.A. - C.N.R.F. 1972.

ARkEz M., Mulıer C. - Comparaison de deux génèrations de sapin (Ahies alta Mill.) : Sı̀ructure génétique de population, hypothèses sur l'action de la sélection naturelle. Annales des Sciences forestières. tome XXVII, fascicule 3, 1970.

Becker W. A. - Manual of procedure in quantitative genetics. Washington State University, 1968.

EutorT G. K. - Wood density in conifers. Technical communication n" 8. Commonwealth Forestry Bureau. Oxford - England, 1970.

Frev K. J., Hokner T. - Heritability in standard units. Agronomy fournal, volume 49, n' 2, fév, 1957.

ILLY G. - Recherches sur lamélioration genétique du pin maritime. Anmales des Sciences foresticres, tome XXIII, fascicule 4, 1966.

KELEeR R. - Des caractéristiques nouvelles pour l'étude des propriétés mécaniques des bois : les composantes de la densité, Amales des Sciences Forestiéres, tome XXV, fascicule 4, 1968.

Krimpl H. - Untersuchungen aber den Drechwuchs bei Fichte. Mitteilungen der forstlichen Bundes Versuchsanstalt. Wien, fascicule 89, 1970.

Larson Ph. R. - Wood formation and the concept of wood quality. New Haven Yale University ; School of Forestry. Bulletin n' 74, 1969.

Namkoovg G.. BAREFoot A. C., Hitchines R. G. - Evaluating control of wood quality through breeding. Tappi, vol. $50, \mathrm{n}^{\circ} 10$, oct. 1969.

Nichous J. W. P., Perry D. H., Hopkins E. R. - Selecting Portuguese Pinus pinaster for Tree Improvement in Australia. - Part 1 : Selection, importation and propagation. Part II ; Wood quality assessment. Ninth British Commonwealth Forestry Conference, 1968.

Nussov B. - Studier av nigra kvalitetsegenskapers genetiska variation hos tall (Pinas silvestris $L_{\text {. }}$ ). Institutionen för skogsgenetik. Skogsh gskolan, $n^{\circ} 3,1968$. Stockholm.

Polge H. - Contribution à l'ètude de la qualité du bois des principales essences résineuses exotiques utilisées dans les reboisements français. Anmales des Sciencess Forextieres, tome XX, fascicule 3, 1963.

Pohge H., ItLy G. - Observations sur l'anisotropie du pin maritime des Landes. Anmales des Sciences Foresticeres, tome 24, fascicule 3, 1967.

Polge H., Ite.y G. - Héritabilité de la densité du bois et corrélation avec la croissance étudiées à l'aide de tests non destructifs sur plants de Pins maritimes de quatre ans. Silvae genetica 17, fascieules 5, 6, sept.-déc. 1968.

Polge H. KelizR R. - Première appréciation de la qualité du bois par utilisation d'un torsiométre. Anmales des Sciences Foresticres, tome 27, fascicule 2, 1970.

Sснмитт J. D. K., Sмrтн W. J. - Wood quality evaluation and improvement in Pinus caribaea Morelet. Queensland Forest Service. Research Note, $n^{\circ} 15$, dèc. 1961. Brisbane.

SмIrн W. J. - The heritability of fibre characteristics and its application in wood quality improvement in forest trees. Silvae genetica, 16, mars-avril 1967.

Sreinhoff R. J., HoFf R. J.- Estimates of the heritability of height growth in western white pine based on parent-progeny relationships. Silvae genetica, 20, juin-juillet 1971. 